\title{
Exploiting convergent evolution to derive a pan-cancer cisplatin sensitivity gene expression signature
}

\author{
Jessica A. Scarborough ${ }^{1,2}$, Steven A. Eschrich ${ }^{3}$, Javier Torres-Roca ${ }^{4}$, Andrew \\ Dhawan ${ }^{5,7, *}$, and Jacob G. Scott ${ }^{1,2,6,8, *}$
}

${ }^{1}$ Systems Biology and Bioinformatics Department, School of Medicine, Case Western Reserve University, Cleveland, $\mathrm{OH}$

${ }^{2}$ Department of Translational Hematology and Oncology Research, Taussig Cancer Institute, Cleveland Clinic, Cleveland, $\mathrm{OH}$

${ }^{5}$ Neurological Institute, Cleveland Clinic, Cleveland, $\mathrm{OH}$

${ }^{3}$ Biostatistics and Bioinformatics Program, Moffitt Cancer Center, Tampa, FL

${ }^{4}$ Department of Radiation Oncology, Moffitt Cancer Center, Tampa, FL

${ }^{6}$ Department of Radiation Oncology, Taussig Cancer Institute, Cleveland Clinic, Cleveland, $\mathrm{OH}$

*Both authors are equal corresponding authors.

7dhawana@ccf.org

${ }^{8}$ scottj10@ccf.org

\begin{abstract}
Precision medicine offers remarkable potential for the treatment of cancer, but is largely focused on tumors that harbor actionable mutations. Gene expression signatures can expand the scope of precision medicine by predicting response to traditional (cytotoxic) chemotherapy agents without relying on changes in mutational status. We present a novel signature extraction method, inspired by the principle of convergent evolution, which states that tumors with disparate genetic backgrounds may evolve similar phenotypes independently. This evolutionary-informed method can be utilized to produce signatures predictive of response to over 200 chemotherapeutic drugs found in the Genomics of Drug Sensitivity in Cancer Database. Here, we demonstrate its use by extracting the Cisplatin Response Signature, CisSig, for use in predicting a common trait (sensitivity to cisplatin) across disparate tumor subtypes (epithelial-origin tumors). CisSig is predictive of cisplatin response within the cell lines and clinical trends in independent datasets of tumor samples. This novel methodology can be used to produce robust signatures for the prediction of traditional chemotherapeutic response, dramatically increasing the reach of personalized medicine in cancer.
\end{abstract}


It is made available under a CC-BY-NC 4.0 International license .

\section{Introduction}

Despite rich collections of cancer "-omic" data, precision medicine research has largely focused on producing therapies that target somatic mutations in proposed driver genes. These therapies have produced some inspiring successes, extending the lives of patients with targetable mutations by months to years. ${ }^{1-3}$ However, the reach of genome-driven care is narrow and most patients without targetable mutations simply have not seen the benefits of personalized medicine. In fact, it was estimated that in 2018 , less than $5 \%$ of cancer patients in the United States could benefit from genome driven care. ${ }^{4}$ Even among the patients who do benefit from genome-driven care, the costs of targeted agents are high and the clinical responses are typically not durable.

Without an actionable mutation, patients often receive conventional cytotoxic chemotherapy. In these scenarios, there are significant opportunities for expanding the reach of precision medicine. For example, gene expression signatures can be used to predict response to these traditional chemotherapy agents without relying on changes in mutational status. Not only is gene expression a powerful measure of phenotype, it is readily translatable to a clinical setting, as patient tumors can undergo RNA-sequencing at relatively low cost and high scale.

Defined as a set of genes (typically fewer than 100), certain gene expression signatures have already been incorporated into standard-of-care and clinical decision-making algorithms (e.g. OncotypeDx ${ }^{5}$, Mammaprint ${ }^{6}$ ). Additionally, signatures of radiosensitivity have been developed and have achieved level 1 evidentiary status for archival tissue. ${ }^{7-10}$ Yet, a major obstacle in the field is finding gene expression signatures that are robust enough to be predictive in novel datasets. And although there is a great need for distilling complex gene expression data into a clinical tool, most published gene expression signatures perform no better than signatures consisting of random genes. ${ }^{11}$ To address this problem, we propose a novel method for the extraction of chemotherapeutic response signatures, utilizing both cell line data, to add isolated drug response information, and tumor sample data, to improve clinical translatability.

As seen in experimental evolution, a variety of evolutionary trajectories can lead to the same phenotype. ${ }^{12-15}$ Figure 1A shows a canonical example of convergent evolution, where genomically disparate species (bats and birds) both evolved the same phenotype of flight independently of one another. Just as bats and birds are genetically closer to mice and reptiles, respectively, individual tumors may be genotypically similar to tumors with differing drug response phenotypes, Figure 1B. Under the selection pressure of a chemotherapeutic agent, tumors may take a wide variety of genomic pathways when evolving drug sensitivity or resistance, meaning that searching for a single genomic marker would be infeasible. In order to understand the basis of chemotherapeutic response, our approach exploits the principles of convergent evolution by combining hundreds of cell lines from a variety of cancer subtypes and extracting transcriptomic patterns of this phenotypic state.
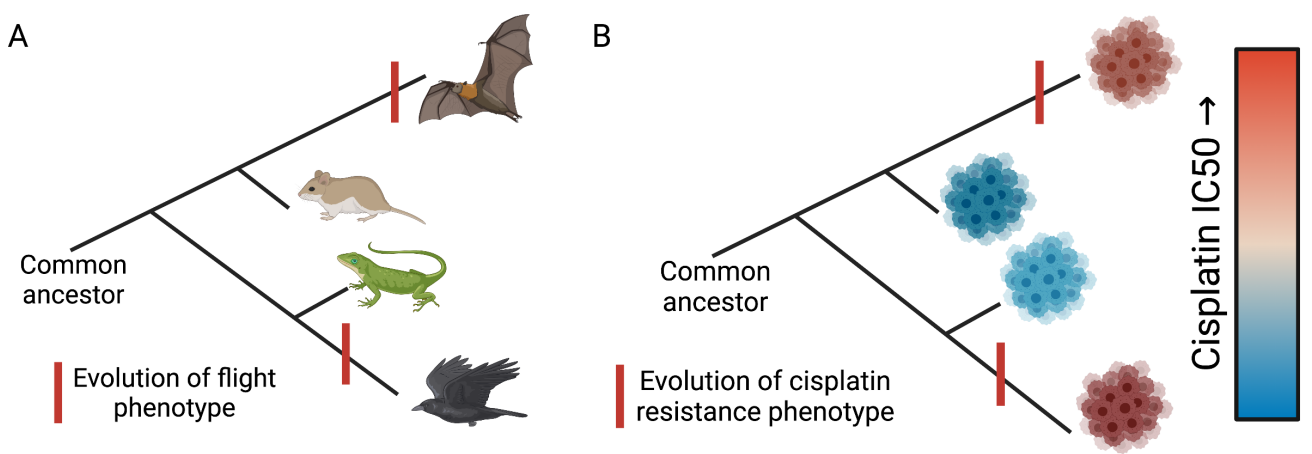

Figure 1. Visual representation of convergent evolution in animals and tumors. A. Birds and bats are genomically disparate, but both have individually evolved the ability to fly. B. Two tumors may evolve cisplatin resistance independently, despite being genomically distinct from one another.

Our work leverages a seed gene approach, as in Buffa et al., where previously identified hypoxia-regulated genes became seeds in a co-expression network, and highly connected genes formed a hypoxia metagene (gene signature) ${ }^{16}$. By extracting genes that are highly co-expressed with biologically significant genes, Buffa et al. produced a robust hypoxia gene signature which was prognostic, even in multivariate analysis and across multiple tissue types.

Our approach empirically derives these seed genes using differential gene expression analysis, comparing cisplatin-sensitive and -resistant cell lines from the Genomics of Drug Sensitivity in Cancer (GDSC) database. The seed genes are then trimmed based on co-expression in epithelial-based tumor samples from The Cancer Genome Atlas (TCGA) ensuring that the final signature contains genes that tend to be expressed together in both cell lines and clinical samples. This novel method may be used to extract gene expression signatures for any quantitative or binary phenotype, and here, we will demonstrate its utility 
with the extraction and validation of the Cisplatin Response Signature (CisSig), for use in predicting response to cisplatin in epithelial-origin tumors (carcinomas). We then show that our final signature is highly predictive of drug response within GDSC cell lines. And finally, we establish that signature expression in independent datasets of clinical tumor samples is congruent with use of cisplatin in standard of care guidelines between disease sites.

\section{Results}

A
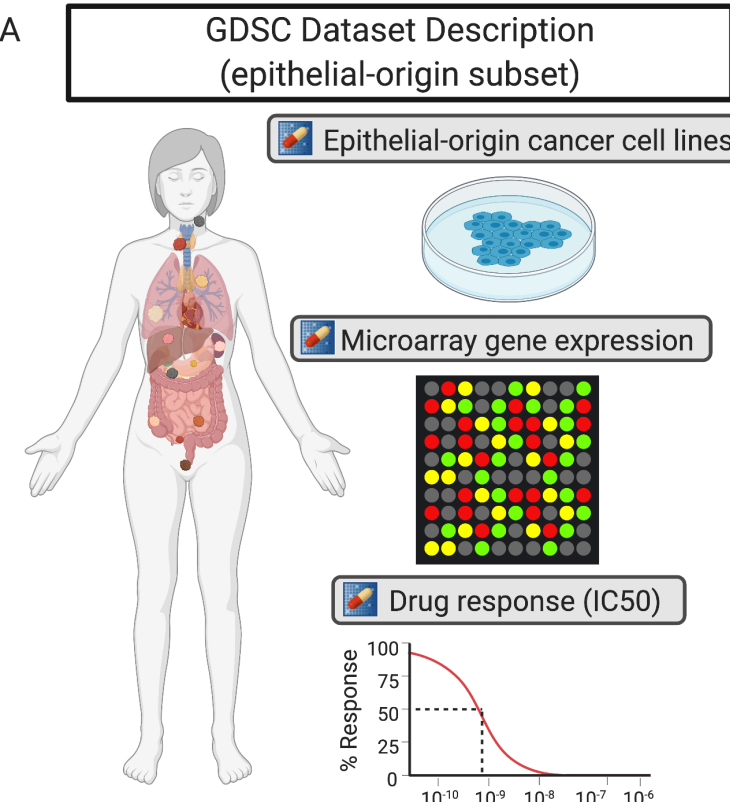

Epithelial-origin cancer cell lines

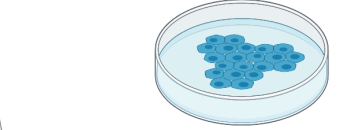

Microarray gene expression

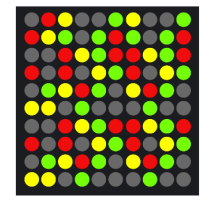

Drug response (IC50)

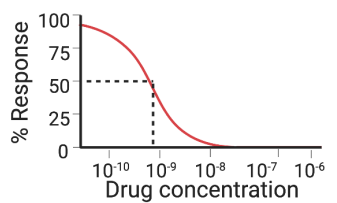

B

Connectivity Gene Extraction Method

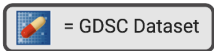

TCGA $=$ TCGA Dataset

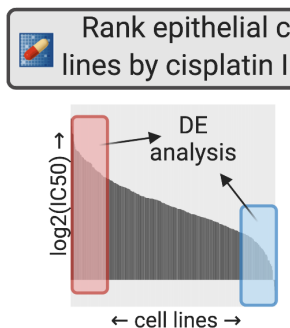

0
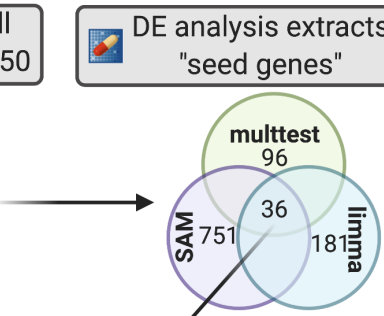
"seed genes"

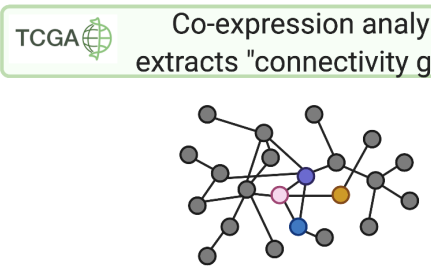

C

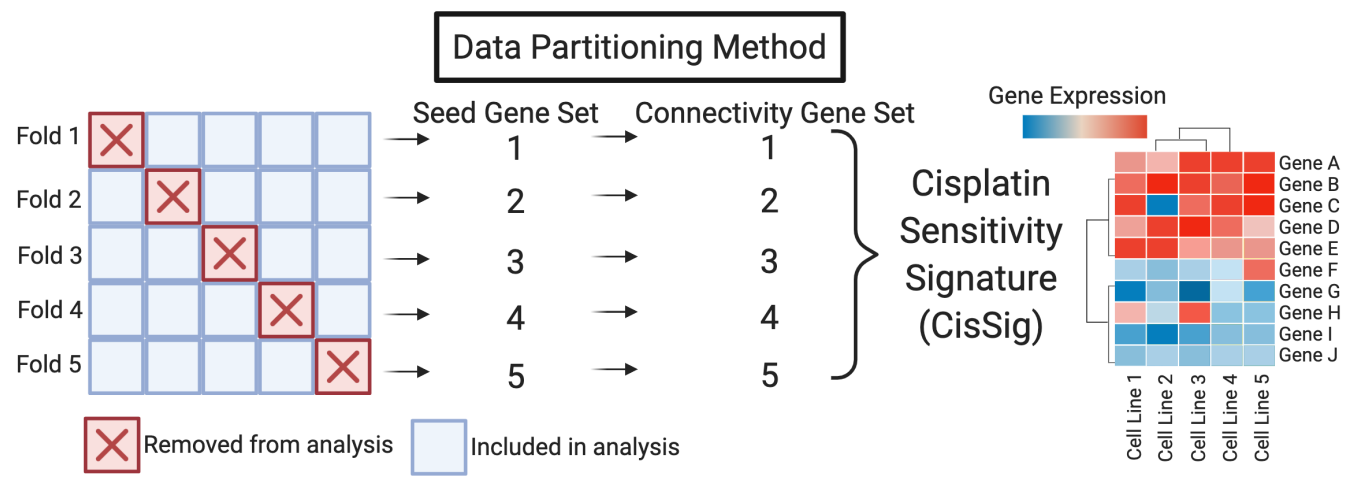

Figure 2. Schematic representation of CisSig derivation. A. Description of the epithelial-origin subset of the Genomics of Drug Discovery in Cancer (GDSC) dataset (denoted with the pill icon in future figures). These data include 429 epithelial-based cancer cell lines, with drug response measurements to over 200 drugs and gene expression characterization via microarray. B. Pipeline for extracting connectivity seeds. First, differential gene expression analysis between the top and bottom $20 \%$ of cisplatin responders found genes with significantly increased expression in a state of cisplatin sensitivity. These differentially expressed genes became "seed genes" in a co-expression network built using gene expression from clinical samples of epithelialbased tumors in The Cancer Genome Atlas (TCGA). Seed genes that were highly co-expressed with each other were denoted as "connectivity genes." C. Schematic of data partitioning, where GDSC epithelial-based cancer cell lines from A. are split into 5 folds. Each fold underwent the pipeline in B. Genes found in at least 3 of the 5 connectivity gene sets were included in the final signature, CisSig.

\section{Convergent evolution informs Cisplatin Response Signature (CisSig) derivation}

CisSig was derived using 429 epithelial-based cancer cell lines in the GDSC Database, each characterized for gene expression and drug response (see Figure 2A). The distribution of disease sites for these cell lines may be found in Supplementary 
Table 2. GDSC gene expression consists of RMA normalized microarray data, details discussed in Methods. This database reports both half-maximal inhibitory concentration (IC50) and area under the drug response curve (AUC) as measures of drug response. A Spearman correlation between these two metrics demonstrated reasonable concordance $(\rho=0.84$, p $<<0.001)$ in measuring cisplatin response for our cell lines of interest (Supplementary Fig. 1). We therefore moved forward with IC50 as the metric of drug response, as it is a more commonly reported measure.

The GDSC epithelial cell lines were partitioned into five folds (each containing 343 or 344 cell lines) with a different $20 \%$ of the cell lines removed, illustrated in Figure 2C. Each of these folds was analyzed with a pipeline of differential gene expression and co-expression analysis, visually depicted in Figure 2B and discussed below. This pipeline was performed across multiple partitions of the data in order to find genes that are consistent between folds, reducing the chance for outlier cell lines to influence the results.

With no pre-filtering of genes, differential gene expression (DE) analysis using limma, ${ }^{17} \mathrm{SAM},{ }^{18}$ and multtest ${ }^{19}$ methods was performed between the top and bottom $20 \%$ of responders (i.e. cell lines with the highest and lowest $20 \%$ of IC50 values). The distribution of disease sites found in each comparison group (resistant and sensitive) for each fold may be found in Supplementary Tables 2-6. More details on parameters and version numbers for each DE method can be found in the Methods section. For each fold, the genes found to be over-expressed in a cisplatin-sensitive state by all three DE methods were termed the "seed genes," resulting in 5 sets of seed genes, as depicted in Figure 2C. Using only intersecting genes between the three methods is done with the goal of increasing stringency by reducing false discovery rate. Results of the DE analysis for each fold are summarized in Supplementary Table 7, and lists of differentially expressed genes from each method, for each fold can be found in Supplementary Data.

A co-expression network was built for each set of seed genes, as described in Methods and visually represented in the bottom panel of Figure 2B. These networks were built using The Cancer Genome Atlas (TCGA) RNA-Seq expression data from epithelial-based tumor samples, comparing the expression of each seed gene and all other genes in the dataset. Seed genes that were highly co-expressed with each other are extracted from each fold, termed "connectivity seeds." Here, we bring in gene expression from tumor samples (not cell lines) to ensure that only genes that are expressed together in both cell lines and tumor samples are included in the final signature. The final gene signature, CisSig, contains any gene found in at least 3 of the 5 sets of connectivity seeds, and the genes included in the signature are listed in Table $\mathbf{1 .}$

Using the 'sigQC' package in R, we analyzed a suite of quality control metrics to assess the robustness of CisSig in a clinical sample (TCGA) dataset. ${ }^{20,21}$ The signature is compared to the 5 sets of seed genes originally extracted from GDSC prior to being refined by co-expression analysis. These results are visualized in a radar plot in Supplementary Figure 2. CisSig demonstrates greater intra-signature correlation, increased correlation between mean and median, and decreased skewness within RNA-expression from TCGA samples of epithelial origin. Other metrics of interest include the coefficient of variance and the proportion $(\sigma)$ of signature genes found in the top $10 \%, 25 \%$ or $50 \%$ of variable genes. These metrics can be used to assess the variability of signature genes within a dataset, where it is ideal to have signature genes that vary more than the background noise. Here, CisSig performs similarly to the unfiltered differential gene expression results. Finally, the these metrics are summarized into a score, also displayed in Supplemental Figure 2, where CisSig outperformed all sets of seed genes.

\section{Increased CisSig expression predicts cisplatin sensitivity within GDSC dataset.}

Figure 3A demonstrates the expression of CisSig genes in cisplatin-sensitive and -resistant GDSC cell lines (top and bottom IC50) quintiles. From this, we see that signature expression tends to be higher (more red) in sensitive, rather than resistant, cell lines. Next, a "CisSig score," the median normalized expression of the 19 CisSig genes, is calculated for the same sensitive and resistant cell lines. The distribution of CisSig score and IC50 among all cell lines can be found in Supplementary Figure 3. Figure 3B shows that sensitive cell lines tend to have higher CisSig scores than resistant cell lines. This is expected, given that the seed genes were initially extracted as genes with increased expression in a cisplatin-sensitive state in the GDSC dataset.

Figure 3C compares the distribution of IC50 between cohorts of GDSC cell lines in this top and bottom quintile of CisSig score. We are terming this plot a "Cell Line Persistence Curve," which resembles a Kaplan-Meier survival curve, but uses IC50 in place of survival time for cell lines. Here, we assume that a cell line does not "survive" when the concentration of cisplatin is greater than it's IC50. For example, at 50\% "survival" on the y-axis, the median IC50 of the high CisSig cohort is $2.76 \mu M$ (left, vertical dashed line), while the median IC50 of the low CisSig cohort is $5.15 \mu M$ (right, vertical dashed line). In other words, cell lines predicted to be resistant (low CisSig) tend to have greater IC50 values and cell lines predicted to be sensitive (high CisSig) tend to have lower IC50 values.

As demonstrated by Venet et al, many published gene signatures do not perform significantly better when predicting survival outcomes than random gene signatures of the same length ${ }^{11}$. Given the large sample size of cell lines, simply testing for statistical significance may not be stringent enough. Therefore, we compared the performance of CisSig's Cell Line Persistence Curve (hazard ratio) to the performance of a null distribution. This null distribution was created using 1000 random gene 
It is made available under a CC-BY-NC 4.0 International license .

Table 1. Genes included in CisSig. These genes all appear in at least 3 of the 5 sets of connectivity seeds.

\begin{tabular}{ll}
\hline HGNC Gene Symbol & Gene Name \\
\hline$A D A T 2$ & Adenosine Deaminase tRNA Specific 2 \\
$A T P 1 B 3$ & ATPase Na+/K+ transporting subunit beta 3 \\
$C D I N 1$ & CDAN1 interacting nuclease 1 \\
C1QBP & Complement C1q binding protein \\
CDC7 & Cell division cycle 7 \\
CDCA7 & Cell division cycle associated 7 \\
FKBP14 & FKBP prolyl isomerase 14 \\
KRT5 & Keratin 5 \\
LRRC8C & Leucine rich repeat containing 8 VRAC subunit C \\
LY6K & Lymphocyte antigen 6 family member K \\
$M M P 10$ & Matrix metallopeptidase 10 \\
NPM3 & Nucleophosmin 3 \\
$P S A T 1$ & Phosphoserine aminotransferase 1 \\
$R I O K 1$ & RIO kinase 1 \\
SLFN11 & Schlafen family member 11 \\
STOML2 & Stomatin like 2 \\
USP31 & Ubiquitin specific peptidase 31 \\
WDR3 & WD repeat domain 3 \\
ZNF750 & Zinc finger protein 750 \\
\hline
\end{tabular}

signatures with the same length as CisSig, assessing the hazard ratio between each signature's Cell Line Persistence Curve. In Figure 3D, we see that CisSig drastically outperforms the top 95\% of this null distribution.

\section{CisSig outperforms the null distributions of drug response prediction models in the GDSC dataset.}

In Figure 3C-D, we demonstrated a novel method to show the stark difference in IC50 distribution for GDSC cell lines with high and low CisSig scores, but it is also important to assess CisSig's predictive power using more traditional methods. To that aim, we built a variety of prediction models using CisSig to predict IC50 as a continuous or binary outcome in epithelial-based GDSC cell lines, described in Table 2. We chose to evaluate the efficacy of using a summary score (CisSig score) in addition to individual gene expression in order to show the value of more "basic" statistical models (e.g. simple linear regression) for producing an easier to interpret model while also gauging the power of using individual CisSig genes in accurately predicting drug response (e.g. random forest). When utilizing expression of each gene individually as the input for our models, we chose a penalized form of regression to prevent overfitting. Finally, for each method selected, we chose to build two models, one with all epithelial-based cell lines and one with only epithelial-based cell lines with high or low signature expression (based on CisSig score quintiles). In doing so, we can gauge whether more extreme expression of CisSig is related to improved drug response prediction accuracy.

In short, simple linear regression models used CisSig score to predict a cell line's IC50 as a continuous variable, while elastic net, L1-, and L2-penalized linear regression models used expression of all CisSig genes to predict a cell line's IC50 as a continuous variable. For these linear regression models, performance was compared using the Spearman correlation coefficient $(\rho)$ between the predicted and actual IC50 value for the cell lines withheld from a given fold's training dataset. The best correlation coefficient between the five folds is chosen to represent each model, shown in Table 2. Simple logistic regression models used CisSig score to predict a cell line's IC50 as a binary outcome (above or below the median), while elastic net-, L1-, and L2-penalized logistic regression, support vector machine (with linear and polynomial kernels), and random forest models were built to use expression of each CisSig gene to predict IC50 as a binary outcome. We used area under the receiver operating characteristic (ROC) curve (AUC) to represent each classification model's performance, again choosing the best of five folds to represent the model in Table 2.

As expected, all models demonstrate improved performance when trained and tested on only cell lines with the highest and lowest signature scores. Additionally, the penalized regression models outperform the simple regression models when comparing the same cell line data inputs. It is expected that including CisSig genes as individual variables would improve performance in comparison to CisSig score, but it is noteworthy that something as simple as median normalized expression of all CisSig genes (also known as the CisSig score) could predict IC50 with the performance shown here.

Figure 4 shows the performance of CisSig for each of the modeling methods described in Table 2. In Figures 4A-B, we 

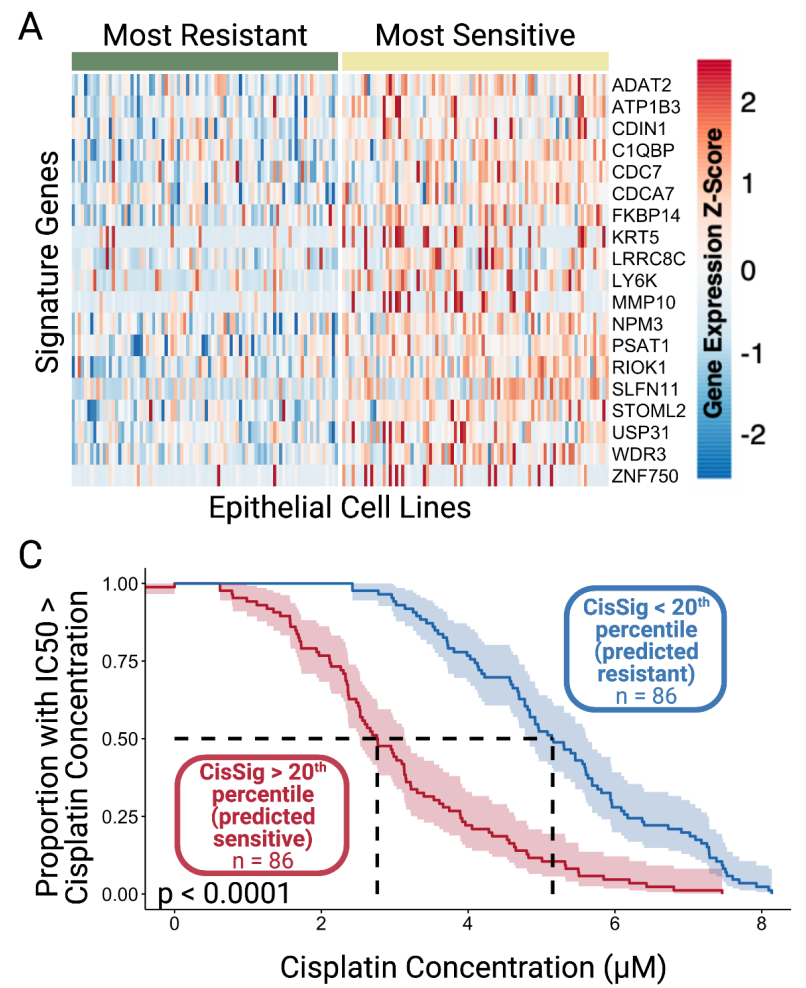

B

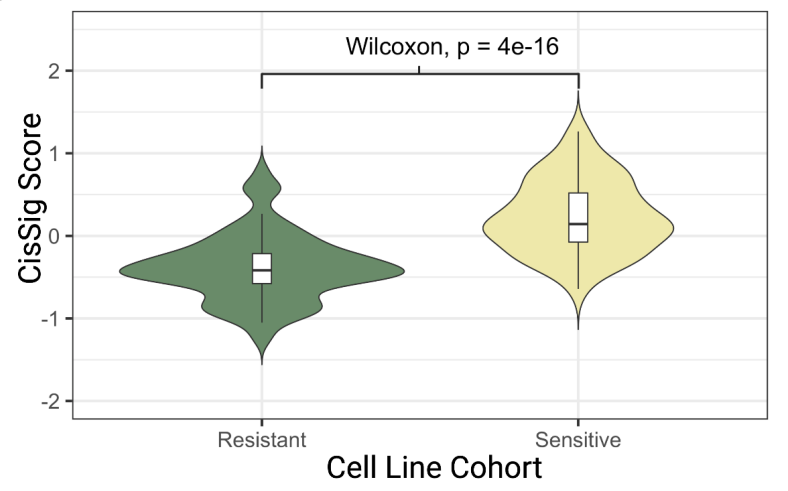

D

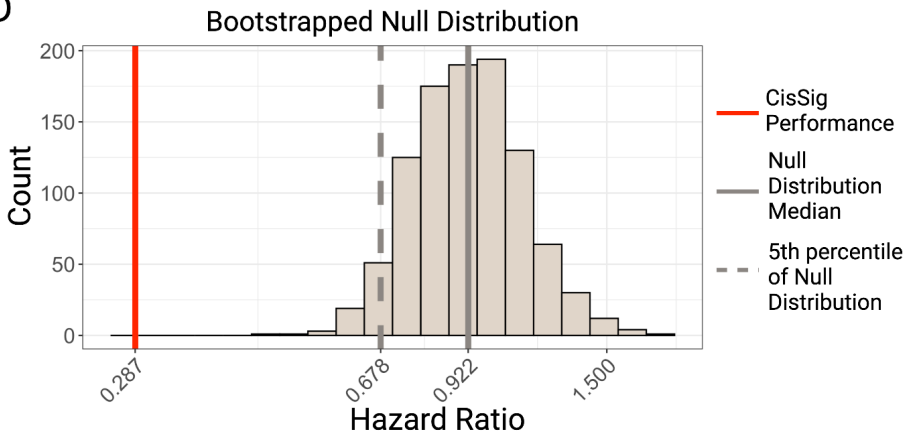

Figure 3. Visualization of CisSig expression within GDSC Dataset. A. An unclustered heatmap showing gene expression of the CisSig genes (rows) in cell lines (columns) from the top and bottom quintiles of cisplatin IC50. Color of the heatmap represents the Z-score of gene expression, normalized to each gene. Cell lines denoted as sensitive (right, yellow bar) tend to display higher expression of CisSig genes than cell lines denoted as resistant (left, green bar). Z-scores above 2.5 are denoted as 2.5, and Z-scores below -2.5 are denoted as -2.5 . B. Violin plots comparing the distribution of CisSig scores between the cell lines in the highest and lowest quintile of cisplatin IC50. A Wilcoxon Rank Sum Test found that the median CisSig scores between these two cohorts was significantly different $(\mathrm{p}<0.001)$. C. Comparison of the distribution cisplatin IC50 between cell lines in the highest and lowest quintile of CisSig score. Y-axis represents the proportion of the cohort with a cisplatin IC50 greater than the cisplatin concentration on the X-axis. A log-rank test between the two cohorts demonstrates significantly different drug response between the two cohorts $(\mathrm{p}<0.0001)$. D. Null distribution of hazard ratio using 1000 random gene signatures with the same length as $\mathrm{CisSig}$ and the model described in $\mathbf{C}$. CisSig's performance is compared to the 95\% confidence interval of the null distribution, where each signature's performance (CisSig and nulls) is represented by the hazard ratio between two cohorts separated by the signature score.

demonstrate how each of the violin plots in Figures 4C-D were built. For example, in Figure 4A, we assess a linear regression model with CisSig score from all epithelial-based GDSC cell lines as the input and IC50 as the continuous outcome. Each model is built with five-fold cross validation, and performance is measured by comparing the predicted and actual IC50 of the testing set using a Spearman correlation. The best performance of the five-folds is used to represent CisSig's performance, shown in Figure 4A. Next, a null distribution, shown in Figure 4B, is produced using 1000 random gene signatures with the same length as CisSig and the same modeling method. Again, the best performance of the five-folds is used to represent each null signature's performance, and CisSig is compared to the null distribution.

We repeated the modeling described in Figures 4A-B for 10 additional modeling methods and the two versions of the dataset (one including all cell lines and another including only cell lines in the top and bottom quintile of signature expression). In Figures 4C-D, we show that CisSig outperforms these null distributions for each of the 11 modeling methods using both versions of the dataset, often outperforming the null distribution altogether. Finally, Supplementary Figures 4-14 presents CisSig's performance in each of the cross validation folds and show a detailed histogram of each model's null distribution.

It is important to note that the wide variety of modeling methods shown here demonstrate that no one method is predictably superior to another, and CisSig shows strong predictive power when using any of them. Models that include only cell lines with more extreme signature expression tend to have improved performance compared to the same modeling method that includes all cell lines. This intimates that more extreme CisSig expression can more accurately predict a cell line's response to cisplatin. 

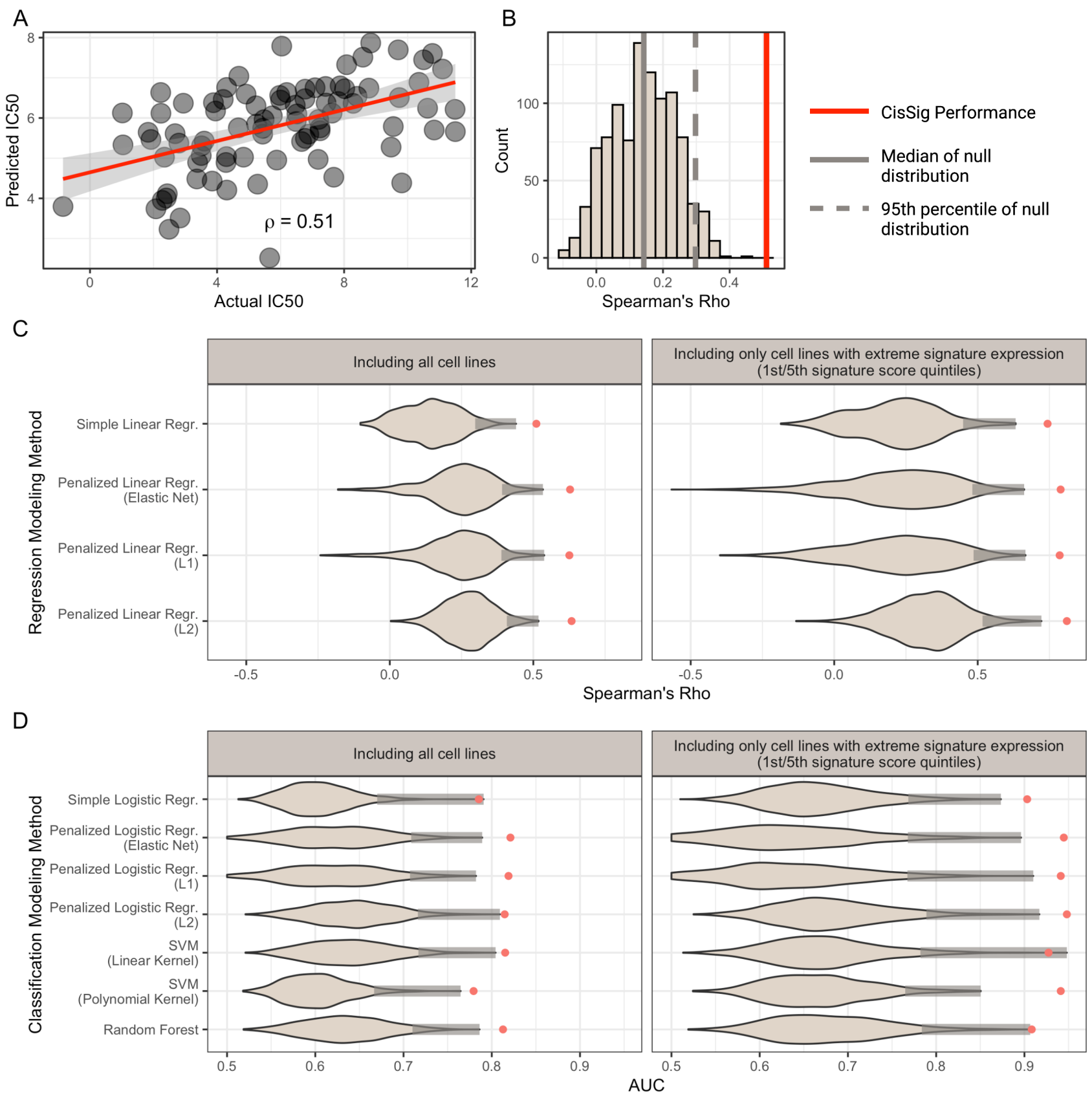

Figure 4. CisSig predicts IC50 using a variety of modeling techniques in the GDSC dataset. A. Scatterplot of the actual vs. predicted IC50 using CisSig score to predict IC50 with linear regression. Plot shows the best performing fold (measured by Spearman's rho) from 5-fold cross validation. B. Null distribution of the performance metric from A. (Spearman's rho), built using 1000 random gene signatures to predict IC50 as described in A. As with CisSig, the metric of the best performing fold is used to represent each null signature. The median of the null distribution and the cutoff for the 95th percentile of the null distribution are represented by the solid and dashed gray line, respectively. CisSig's performance, red solid line, outperforms at least $95 \%$ of the null distribution. C. Violin plots containing the null distribution of performance metrics for 11 modeling methods. Each distribution was created as discussed in A-B, where CisSig's performance is compared to the performance of 1000 random gene signatures of the same length. For each violin, a shaded gray bar represents the top 5\% of each null distribution and CisSig's performance is shown with a red dot. The modeling methods, including input and output, are described in Table 2. 
It is made available under a CC-BY-NC 4.0 International license .

Table 2. Model details and validation results for the prediction of cisplatin response using CisSig in GDSC dataset.

\begin{tabular}{|c|c|c|c|c|c|}
\hline Input & Output & Method & Included Data & Metric & Value \\
\hline CisSig Score & IC50 (continuous) & Simple Linear Regression & All & Corr. Coef. & 0.51 \\
\hline CisSig Score & IC50 (continuous) & Simple Linear Regression & Quintiles & Corr. Coef. & 0.74 \\
\hline All gene expression & IC50 (continuous) & Elastic Net Linear Regression & All & Corr. Coef. & 0.63 \\
\hline All gene expression & IC50 (continuous) & Elastic Net Linear Regression & Quintiles & Corr. Coef. & 0.79 \\
\hline All gene expression & IC50 (continuous) & L1 Linear Regression & All & Corr. Coef. & 0.63 \\
\hline All gene expression & IC50 (continuous) & L1 Linear Regression & Quintiles & Corr. Coef. & 0.79 \\
\hline All gene expression & IC50 (continuous) & L2 Linear Regression & All & Corr. Coef. & 0.63 \\
\hline All gene expression & IC50 (continuous) & L2 Linear Regression & Quintiles & Corr. Coef. & 0.81 \\
\hline All gene expression & IC50 (binary) & Simple Logistic Regression & All & AUC & 0.79 \\
\hline All gene expression & IC50 (binary) & Simple Logistic Regression & Quintiles & AUC & 0.90 \\
\hline All gene expression & IC50 (binary) & Elastic Net Logistic Regression & All & AUC & 0.82 \\
\hline All gene expression & IC50 (binary) & Elastic Net Logistic Regression & Quintiles & AUC & 0.94 \\
\hline All gene expression & IC50 (binary) & L1 Logistic Regression & All & AUC & 0.82 \\
\hline All gene expression & IC50 (binary) & L1 Logistic Regression & Quintiles & AUC & 0.94 \\
\hline All gene expression & IC50 (binary) & L2 Logistic Regression & All & AUC & 0.81 \\
\hline All gene expression & IC50 (binary) & L2 Logistic Regression & Quintiles & AUC & 0.95 \\
\hline All gene expression & IC50 (binary) & SVM (linear kernel) & All & AUC & 0.82 \\
\hline All gene expression & IC50 (binary) & SVM (linear kernel) & Quintiles & AUC & 0.93 \\
\hline All gene expression & IC50 (binary) & SVM (polynomial kernel) & All & AUC & 0.78 \\
\hline All gene expression & IC50 (binary) & SVM (polynomial kernel) & Quintiles & AUC & 0.94 \\
\hline All gene expression & IC50 (binary) & Random Forest & All & AUC & 0.81 \\
\hline All gene expression & IC50 (binary) & Random Forest & Quintiles & AUC & 0.91 \\
\hline
\end{tabular}

\section{Ranking cancer subtypes by CisSig expression is concordant with observed clinical trends.}

The consistently strong validation statistics displayed in Figures $\mathbf{3}$ and $\mathbf{4}$ demonstrate that this novel signature extraction methodology is capable of selecting genes with strong predictive power within the source dataset. In other words, it is a powerful tool for feature selection. In order to assess translation into novel datasets; however, predictive power must be demonstrated in datasets that were not used to select genes of interest.

Using three large datasets, we assessed how expression of CisSig relates to cisplatin use across epithelial-based cancer disease sites. CisSig score was calculated for all samples (cell lines or clinical tumor samples) in GDSC, TCGA, and Total Cancer Care (TCC) databases. In order to visualize these scores on a log-transformed axis, signature score was linearly scaled, such that the lowest score became exactly 1 .

In Figure 5, disease sites were ranked by the median signature score for the cohort in GDSC (left), TCGA (middle), and TCC (right) datasets. Furthermore, each disease site is labeled as utilizing cisplatin in NCCN treatment guidelines (green circle), using cisplatin in very select circumstances (yellow bars), or not having cisplatin included in NCCN treatment guidelines (red square). In all datasets, we see that disease sites with higher CisSig scores tend to have cisplatin included in treatment guidelines, while those with lower scores tend to not have cisplatin included in treatment guidelines.

Finally, disease site rank was compared between datasets using Spearman's correlation. There is a strong correlation between the rank of shared disease sites of all three datasets. Between GDSC and TCGA, Spearman's $\rho$ is $0.77(\mathrm{p}<0.001)$. Between GDSC and TCC, Spearman's $\rho$ is $0.92(\mathrm{p}<0.001)$. And between TCGA and TCC, Spearman's $\rho$ is $0.93(\mathrm{p}<0.001)$. This high degree of concordance between datasets signifies that CisSig displays consistent expression between a variety of data sources (including between microarray and RNA-seq methods).

\section{Discussion}

The principles of convergent evolution tell us that genetically distant organisms can evolve similar traits in order to become more fit under the same selection pressure. In cancer, therefore, we cannot ignore the possibility that different mutations may lead to the same phenotype. Therefore, our novel method groups convergent phenotypes and uses expression profiling to better predict drug response in cancer. In doing so, we harnessed the power of over 400 epithelial-origin cell lines in the GDSC Database to extract CisSig, a gene expression signature for use in predicting cisplatin response in epithelial-origin tumors.

Gene expression signatures can expand the reach of precision medicine to impact the vast majority of patients whose tumors 


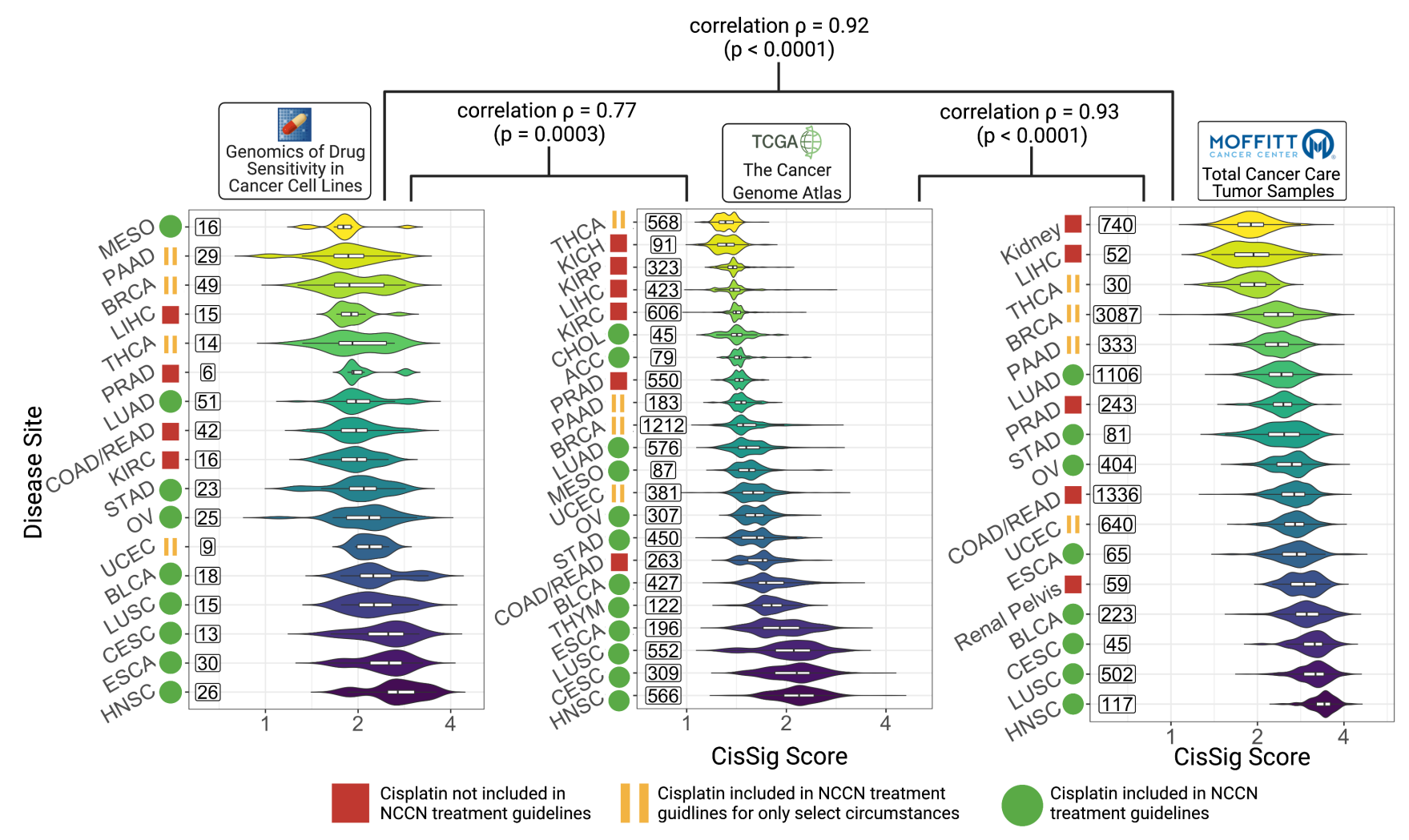

Figure 5. Cancer subtypes with greater CisSig expression tend to have cisplatin included in standard of care guidelines. Cancer subtypes are ranked by median CisSig Score in three data sets, GDSC (left), TCGA (middle), and TCC (right). The color of each violin plot represents the rank of the cancer subtype. The ranks of intersecting subtypes between each dataset are compared with Spearman's rank correlation, reported with correlation $\rho$ and p-value. Rank correlation $\rho$ between GDSC and TCGA and GDSC and TCC datasets is $0.77(\mathrm{p}=0.0003)$ and $0.902(\mathrm{p} « 0.0001)$. Rank correlation $\rho$ between TCGA and TCC datasets is 0.93 ( $\mathrm{p}$ « 0.0001). Violin plots display the distribution of CisSig scores for each cancer subtype. Within each violin, a boxplot denotes median signature score for each subtype (middle horizontal line) and 25th/75th percentile for signature scores (box edges). Numbers to the left of each violin plot represent sample size included in each cancer subtype.

do not harbor actionable mutations. Yet, finding signatures with significant translational potential remains difficult. This is in part because although cell lines are preferable for high throughput analysis, they tend to demonstrate some divergence from their tumors of origin. 22,23

As demonstrated by many predictive modeling methods, our gene signature is highly effective at predicting drug response in GDSC cell lines, from which it was originally derived. This initial validation is an important step, but demonstrating utility with independent clinical samples is crucial for assessing the translational potential of our signature. Unlike with cell lines, high throughput characterization of drug response (i.e. IC50, AUC, etc) in clinical tumor samples is not feasible. ${ }^{22}$ Because of this, many researchers use survival as a surrogate measure of treatment response for tumor samples. However, without a known clinical history of cisplatin treatment, we cannot use survival as a surrogate measure of cisplatin response.

As such, we chose to assess how well CisSig expression in tumor samples correlates to clinical treatment trends. With this analysis, we show that cancer subtypes frequently treated with cisplatin (e.g. head and neck, cervical) tend to have greater CisSig scores in GDSC, TCGA, and TCC datasets. GDSC was directly used in the extraction of CisSig and TCGA is used only for co-expression analysis in trimming the signature genes, but the TCC database was not used in any part of the extraction methodology. And although predictive modeling within this independent dataset is not feasible, this result does show that expression of CisSig tends to be congruent with current clinical practices, an indication that CisSig has translational potential.

This signature extraction method is, of course, not without limitations. First, a single tumor sample may not capture the intratumoral heterogeneity that is crucial for predicting the physiological response to a drug. Next, although the signature was extracted to find genes with importance across pan-cancer (epithelial-based) tumor subtypes, clinical validation must occur within individual disease sites. Given the heterogeneity between tumor subtypes, disease-site specific versions of CisSig may 
A

A

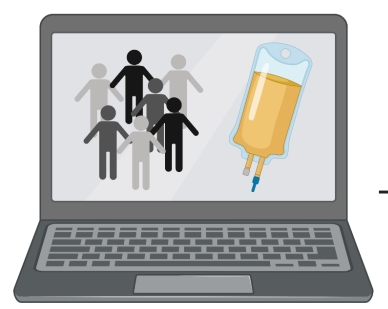

Retrospective Trial Design

Archival tissue from

clinical trial

(single disease site)

Repeat at least once with independent dataset
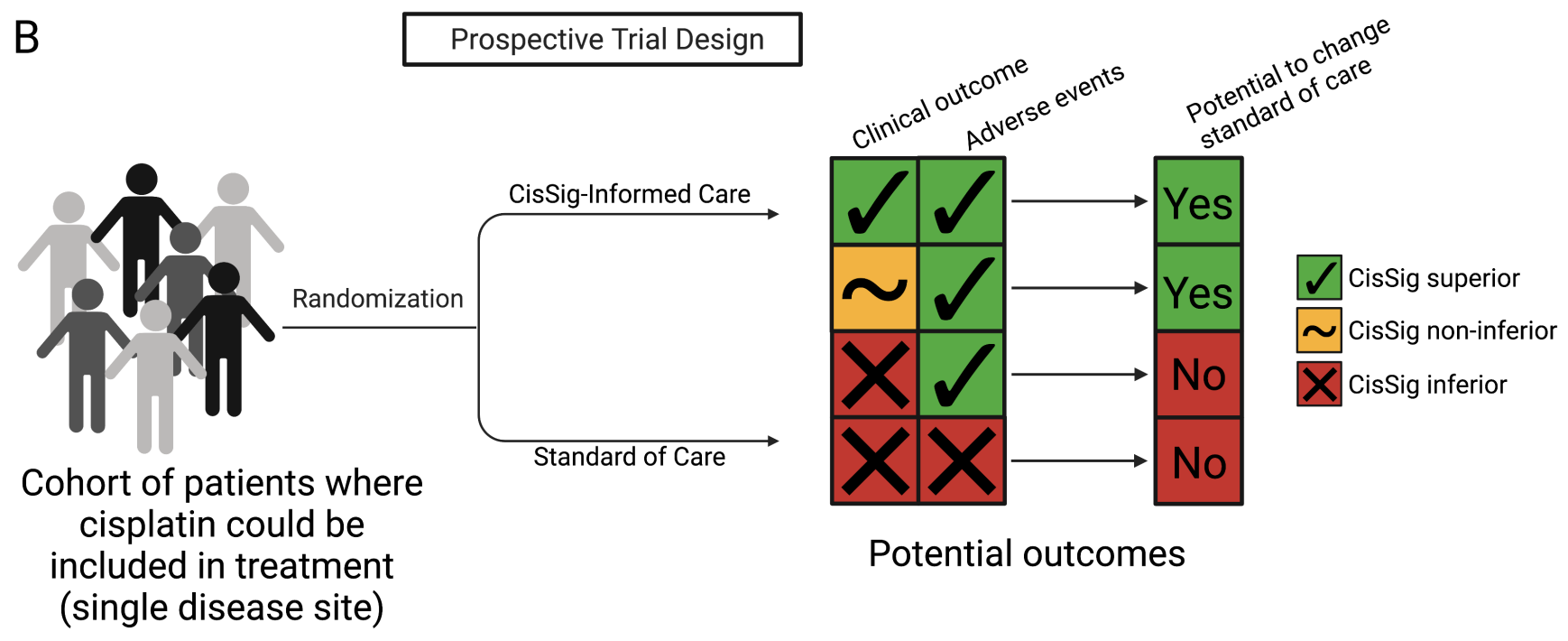

Potential outcomes

Figure 6. Pathways for CisSig to reach level 1 evidence. A. Retrospective trial design. Measure CisSig expression in archival tissue of a single disease site from a prior clinical trial. Determine if cohorts of high vs. low CisSig expression have significantly different survival trends. Repeat at least once with an independent dataset to reach level 1 evidence. B. Prospective trial design. Begin with a cohort of patients with a single cancer subtype where cisplatin may or may not be included in their treatment plan. Patients are randomized into a CisSig-Informed Cohort or a Standard of Care Cohort. In the CisSig-Informed Cohort, clinicians will be informed on CisSig expression and what it means regarding predicted therapeutic response. They will use this information when counseling the patient in deciding between therapeutic options. The Standard of Care Cohort will not receive any information regarding a patient's CisSig expression. The two cohorts will be compared regarding clinical outcome, adverse events, and other factors. A variety of differences between the two cohorts could lead to level 1 evidence for the use of CisSig in that disease site.

require trimming the genes of this pan-cancer signature even further. Additionally, as discussed previously, using cell line expression data as the basis of a clinical signature is necessary given the current limitations of high throughput databases, but it can hinder translation. Therefore, a key future direction will be testing the signature in clinical data to determine if patient response to cisplatin can be stratified by signature expression.

Figure 6 shows two pathways that could demonstrate the successful clinical translation of CisSig, providing level 1 evidence for its use. First, a retrospective trial design, displayed in Figure 6A, could take archival tissue from a clinical trial of an epithelial-based disease site (e.g. squamous lung cancer, cervical cancer, etc.) where all patients have undergone cisplatin-containing treatment. After CisSig expression is measured for all samples, patients will be separated into cohorts of high and low CisSig expression, where it is predicted that the high CisSig cohort will have improved survival. According to Burns, et $\mathrm{al}^{24}$, this retrospective trial design must be completed with at least two independent datasets to reach level 1 evidence.

Next, Figure 6B presents a prospective trial design for assessing the utility of CisSig in a clinical setting. Starting with 
a cohort of patients where cisplatin could be included in the treatment plan, patients would be randomized into the CisSiginformed care or standard of care treatment cohort. With CisSig-informed care, clinicians will be informed of the patient's predicted response to cisplatin and encouraged to utilize this information when determining whether cisplatin should be included in the patient's treatment plan. There are many possible outcomes of this trial design, a few of which are demonstrated in Figure 6B. Ideally, CisSig-informed care will improve both survival outcomes and adverse events. Yet, there would still be level 1 evidence to support the use of CisSig even if survival outcomes were non-inferior, given that adverse events are improved in the CisSig-informed care cohort.

Selection, like drug treatment acts on phenotype. And in this work, we demonstrate a novel gene signature extraction method-informed by principles of convergent evolution-where we find shared transcriptomic markers of drug response phenotype in tumors that appear genotypically disparate. By harnessing the power of a large dataset, such as the GDSC, we extracted a biologically-inspired product, CisSig. Expanding this method to produce signatures for response prediction to a variety of chemotherapeutic agents will lead to a monumental expansion of precision medicine in cancer.

\section{Methods}

\section{Data Collection and Pre-Processing}

All data cleaning, analysis, and plotting was performed using R (Version 4.0.5) with RStudio.

\section{GDSC Gene Expression Data}

Microarray mRNA expression, drug response, and meta-data for 983 cell lines and 251 drugs was downloaded from the Genomics in Drug Sensitivity Database (GDSC) ${ }^{25}$. The expression and meta-data were last updated 4 July 2016. The GDSC database can be accessed at https://www.cancerrxgene.org/. Documentation for the GDSC database states that the RMA normalized ${ }^{26,27}$ expression data for all cell lines were collected via Human Genome U219 96-Array Plate using the Gene Titan MC instrument (Affymetrix). Further the robust multi-array analysis (RMA) algorithm was used to normalize the data, reporting intensity values for 18562 individual loci. The raw data and probe ID mappings were deposited in ArrayExpress (accession number: E-MTAB-3610). The RMA processed dataset is available at http://www.cancerrxgene.org/gdsc1000/.

Epithelial-based cell lines are extracted based on the following GDSC tissue descriptors (exact labels found in database): head and neck, oesophagus, breast, biliary_tract, large_intestine, liver, adrenal_gland, stomach, kidney, lung_NSCLC_adenocarcinoma, lung_NSCLC_squamous-_cell_carcinoma, mesothelioma, pancreas, skin_other, thyroid, Bladder, cervix, endometrium, ovary, prostate, testis, urogenital_system_other, uterus.

\section{GDSC Drug Response Data}

The drug response data in the GDSC database was last updated 27 March 2018; this version is referred to as "GDSC2." Cisplatin drug concentration is reported in $\mu M$. Raw viability data were processed using the R package, gdscIC50, where they were normalized with negative controls (media alone) and positive controls (media only wells with no cells). Dose-response curves were fit using a multi-level fixed effect model with a classic sigmoidal curve shape assumed. This model was fitted using all cell line/drug combinations that were screened instead of fitting separate models to individual drug-response series. In this approach, the shape parameter only changes between cell lines, but the position parameter is adjusted between cell lines and compounds. Additional information regarding dose-response curve fitting may be found at Vis et al. ${ }^{28}$. Fitting models to all dose-response series leads to improved robustness for more accurate IC50 and AUC estimates.

\section{TCGA Gene Expression Data}

RNA-Seq by Expectation Maximization (RSEM) normalized gene expression for epithelial-based cancers was downloaded from The Cancer Genome Atlas (TCGA) database, which was accessed through the Firebrowse database using the 'RTCGAToolbox' package (version 2.20.0) ${ }^{29}$ in R. The following TCGA Study Abbreviations were downloaded (exact labels found in database): ACC, BLCA, BRCA, CESC, CHOL, COADREAD, ESCA, HNSC, KIRC, KIRP, KICH, LIHC, LUAD, LUSC, MESO, OV, PAAD, PRAD, STAD, THCA, THYM, UCEC. These values were measured through the Illumina HiSeq RNAseq V2 platform and were $\log 2$ transformed.

\section{Total Cancer Care (TCC) Gene Expression Data}

The Total Cancer Care Dataset is collected by the H. Lee Moffitt Cancer Center and Research Institute using protocols described in Fenstermacher et al ${ }^{30,31}$. The Total Cancer Care (TCC) protocol is a prospective tissue collection protocol that has been active at Moffitt Cancer Center (Tampa, FL, USA) and 17 other institutions since 2006. We assayed tumours from adult patients enrolled in the TCC protocol on Affymetrix Hu-RSTA-2a520709, which contains approximately 60,000 probesets representing 25,000 genes. Chips were normalised using iterative rank-order normalisation. ${ }^{32}$ Batch effects were reduced using partial-least squares. We extracted from the TCC database normalised, debatched expression values for 9,063 samples from 17 sites of 
epithelial origin and the 19 CisSig genes. We excluded all metastatic duplicate samples and disease sites with fewer than 25 samples.

\section{Drug Response Quality Control}

IC50 is an imperfect measure of drug response, yet it is widely used throughout the literature. It is defined as the concentration of drug at which cells experience 50\% inhibitory effect. Another measure of drug response is area under the drug response curve, which is defined as the integral of a drug response curve, where cellular activity is measured on the y-axis and drug concentration is measured on the x-axis. IC50 and AUC values for all epithelial cell lines are compared using a Spearman correlation test (see Supplementary Figure 1) in order to assess concordance between the two metrics.

\section{Differential Gene Expression Analysis}

As seen in Figure 2C, the GDSC dataset is split into 5-folds, where $20 \%$ of the cell lines are removed from further analysis for each of the 5 runs. This leaves 343 or 344 cell lines in each of the 5 partitions. After data partitioning, the top $20 \%$ and bottom $20 \%$ are extracted for comparison using differential expression analysis, Figure $\mathbf{2 C}$.

Differential expression analysis is performed using three algorithms: significance analysis of microarrays (SAM), resampling-based multiple hypothesis testing, and linear models for microarrays (limma), which are implemented using R packages 'samr' 18 (version 3.0), 'multtest' ${ }^{19}$ (version 2.46.0), and 'limma'17 (version version 3.46.0), respectively. Gene expression was pre-normalized using RMA (discussed above) and genes were not pre-filtered before this analysis. This analysis has 69 samples per group, which is appropriate given the demonstration by Baccarella et al. showing that differential expression results begin to vary problematically beginning when there are as few as 8 samples per group ${ }^{33}$.

A false discovery rate or p-value cutoff of 0.20 was chosen for each method. The 'samr' and 'multtest' method were both set to the same seed. The "samr' method used 10,000 permutations (parameter: "nperm") and test statistic was set to "standard" for t-test (parameter: “testStatistic"). The 'limma' method used no p-value adjustment method (parameter: "adjust.method") and a log-fold change cutoff of 0.5 (parameter: "lfc"). The 'multtest' method used 1,000 bootstrap iterations (parameter: "B") and single-step minP for multiple testing procedure (parameter: "method"). All other parameters for the three algorithms were set to default. The intersection of the genes found to have significantly increased expression in sensitive cell lines by the three algorithms is termed "seed genes" for use in future co-expression analysis. An FDR cutoff of 0.2 is a relatively non-stringent FDR cutoff; it was chosen in order to include a variety of genes before taking the intersection of results between the three methods.

\section{Co-Expression Network Analysis and Final Signature Derivation}

The co-expression network, represented in the pipeline of Figure 2B, is made by performing a pairwise Spearman correlation between the expression of each seed gene and every other gene (including other seed genes) except itself. The correlation coefficient for each pairwise comparison is termed the "affinity score." Next, the network is transformed so that the largest $5 \%$ of affinity scores are transformed to 1 and all other scores become 0 . This is done without squaring the scores in order to extract only positive correlations. The average affinity score for each gene compared to each seed gene is then derived; this value becomes known as a gene's "connectivity score." The intersection between the differentially expressed seed genes and genes with the top $20 \%$ of the highest connectivity scores become known as the "connectivity genes." Five sets of connectivity genes are compiled, one for each data partition. The final signature (CisSig) is produced by extracting any gene that is found in at least three of the five connectivity gene sets.

\section{Signature Quality Control in TCGA}

In order to examine how CisSig compares to the original differential gene expression results and ensure portability to novel datasets, we perform a quality control analysis within the TCGA dataset using the 'sigQC' R package ${ }^{20}$ with methodology as in Dhawan et al. $2019^{21}$. Here, various metrics are calculated using the expression of the genes found in the gene expression signature and the 5 sets of differential expression analysis results. These metrics include intra-signature correlation, correlation between the mean expression and first principal component, and skewness of the signature expression. The final results of all the metrics calculated for each signature are displayed in a radar plot, with a summary score of each set of genes (signature) tested. This summary score is the ratio of the area within the radar plot and the full polygon if each metric was the highest value possible.

\section{Predicting cell line IC50 using CisSig in GDSC}

A cell line or sample's median normalized expression value of the CisSig genes is termed the CisSig score. Cell lines were again organized into five folds (independent of the data partitioning used in the signature extraction, described in Figure 2C). Predictive models were built using $80 \%$ of the cell lines (training cell lines) and tested on the $20 \%$ of the cell lines withheld from the model (validation cell lines). All models were built with two versions of input-one using all of the epithelial-based 
cell lines in the GDSC database and the other using only the cell lines in the top and bottom quintiles of CisSig score. When using all the epithelial-based cell lines, training sets consist of 344-345 cell lines, while testing sets consist of 86 cell lines. When using only the cell lines in the top and bottom quintiles for signature expression, training sets consist of 137 or 138 cell lines and testing sets consist of 34 or 35 cell lines.

Simple linear and logistic regression was used to predict IC50 as a continuous variable with CisSig score as the input. Elastic net, L1-, and L2-penalized linear regression methods utilized the expression of each of the 19 CisSig genes to predict IC50 as a continuous variable. Elastic net, L1-, and L2-penalized logistic regression methods, support vector machine (SVM), and random forest methods utilized expression of each of the 19 CisSig genes to predict IC50 as a binary variable (above or below the median of the group). All linear regression models were evaluated using the Spearman correlation coefficient between true and predicted IC50 values from the validation set. Classification models (logistic regression, SVM, and random forest) were evaluated using area under the receiver operating characteristic (ROC) curve (AUC).

Elastic net, L1-, and L2-penalized linear and logistic regression models were built using the 'glmnet' package (version 4.1-2) in R. The alpha parameter was set to 0.5, 1, and 0 for elastic net, L1-, and L2-penalized regression, respectively. Models were tuned with 10 -fold cross validation to choose a value for lambda with the best predictive capabilities based on mean square error for linear models and misclassification error for logistic models.

SVM models were built with the 'e1071' package (version 1.7-8) in R, using both a linear and polynomial kernel. Models were tuned with 10-fold cross validation to choose the best value for degree (from 3,4 , 5), gamma (from $10^{-3}, 10^{-2}, 10^{-1}, 1,10^{1}$, $10^{2}, 10^{3}$ ), and cost (from $10^{-3}, 10^{-2}, 10^{-1}, 1,10^{1}, 10^{2}, 10^{3}$ ).

Random forest models were built with the 'randomForest' package (version 4.6-14), and each model grew 500 trees. All other parameters in training the prediction models were default.

\section{Cell Line Persistence Curves}

Cell lines with high CisSig scores (predicting the more sensitive cell lines) and low signatures scores (predicting the more resistant cell lines) are separated by quintile. A Kaplan-Meier survival model is built for the two cohorts using IC50 in lieu of survival time. A log-rank test compares the two survival curves to analyze if the two cohorts of signature expression are related to different "survival" of higher IC50s in each group.

\section{Null distributions of cell line IC50 models}

CisSig's performance was compared to a null distribution for all models built, including all models used to predict IC50 as a continuous or binary variable and the cell line persistence models using the log-rank test to compare the two survival curves. To build each null distribution, 1000 random gene signatures with the same length as CisSig were chosen. Each random gene signature was selected using all genes included in the GDSC expression profiling without replacement. The performance of each random signature was tested in each individual modeling method, producing a null distribution for each modeling method.

As discussed above, the predictive models utilize five-fold cross validation and the best summary statistic of the five folds is chosen to represent the signature's performance. This remains consistent for the null models, where the best summary statistic of the five folds is used to represent each random signature. Again, all code for building the testing and null models may be found in the GitHub repository listed in Code and Data Availability.

\section{Ranking disease sites in GDSC, TCGA, and TCC by CisSig Score}

All epithelial-origin cell lines or tumor samples in the GDSC, TCGA, and TCC datasets had CisSig Score calculated as previously described. For the purposes of plotting on a log-scale, the scores were linearly adjusted by adding the absolute value of the lowest score plus 1 to each sample's score, making the lowest score now 1. For example, if the lowest signature score for the dataset was $-5,6$ was added to each sample's score. Disease sites within each dataset were ranked by median CisSig score. For disease sites shared between datasets, a Spearman correlation was performed to assess how the rank of disease sites compare between datasets.

\section{Classifying disease sites by cisplatin use}

NCCN Treatment Guidelines for each disease site were manually searched, versions listed in Supplementary Table 8. Disease sites were classified as including cisplatin in treatment guidelines, only including cisplatin in very select circumstances, or not including cisplatin in treatment guidelines. For those classified as only using cisplatin in select circumstances, details are noted in Supplementary Table 8.

\section{Data and code availability}

The code to download all data, extract CisSig, perform validation of the signature, and reproduce all figures in the manuscript is available via GitHub at

https://github.com/jessicascarborough/cissig. 
medRxiv preprint doi: https://doi.org/10.1101/2021.11.10.21265799; this version posted November 11, 2021. The copyright holder for this preprint (which was not certified by peer review) is the author/funder, who has granted medRxiv a license to display the preprint in perpetuity.

It is made available under a CC-BY-NC 4.0 International license .

355

\section{Acknowledgements}

J.G.S. would like to thank NIH (5R37CA244613-02) and the American Cancer Society (RSG-20-096-01) for their generous support. J.A.S. thanks the NIH for their support through the T32GM007250 and 1F30CA257076-01 grants. The results published here are in whole or part based upon data generated by the TCGA Research Network: https://www.cancer.gov/tcga. All authors are grateful to the cancer patients who provided tissue for further study in the GDSC, TCGA, and TCC datasets. This work made use of the High Performance Computing Resource in the Core Facility for Advanced Research Computing at Case Western Reserve University.

\section{Author contributions}

J.A.S. contributed to experimental design, wrote all associated code, analyzed data, and wrote the manuscript. A.D. contributed to experimental design and analyzed data. S.A.E. analyzed data. J.T.R. contributed to experimental design. J.G.S. contributed to experimental design, analyzed data, and wrote the manuscript. All authors read and approved of the manuscript. 


\section{References}

1. Hirsch, F. R. et al. Lung cancer: current therapies and new targeted treatments. The Lancet 389, 299-311 (2017).

2. Solomon, B. J. et al. First-line crizotinib versus chemotherapy in alk-positive lung cancer. New Engl. J. Medicine 371, 2167-2177 (2014).

3. Prasad, V., De Jesus, K. \& Mailankody, S. The high price of anticancer drugs: origins, implications, barriers, solutions. Nat. reviews Clin. oncology 14, 381 (2017).

4. Marquart, J., Chen, E. Y. \& Prasad, V. Estimation of the percentage of us patients with cancer who benefit from genome-driven oncology. JAMA oncology 4, 1093-1098 (2018). PMCID: PMC6143048.

5. Sparano, J. A. et al. Adjuvant chemotherapy guided by a 21-gene expression assay in breast cancer. New Engl. J. Medicine 379, 111-121 (2018).

6. Soliman, H. et al. Mammaprint guides treatment decisions in breast cancer: results of the impact trial. BMC cancer 20, 81 (2020).

7. Scott, J. G. et al. A genome-based model for adjusting radiotherapy dose (gard): a retrospective, cohort-based study. The lancet oncology 18, 202-211 (2017).

8. Scott, J. G. et al. Pan-cancer prediction of radiotherapy benefit using genomic-adjusted radiation dose (gard): a cohort-based pooled analysis. The Lancet Oncol. 22, 1221-1229 (2021).

9. Eschrich, S. A. et al. A gene expression model of intrinsic tumor radiosensitivity: prediction of response and prognosis after chemoradiation. Int. J. Radiat. Oncol. Biol. Phys. 75, 489-496 (2009).

10. Torres-Roca, J. F. A molecular assay of tumor radiosensitivity: a roadmap towards biology-based personalized radiation therapy. Pers. medicine 9, 547-557 (2012).

11. Venet, D., Dumont, J. E. \& Detours, V. Most random gene expression signatures are significantly associated with breast cancer outcome. PLoS computational biology 7, e1002240 (2011).

12. Nichol, D. et al. Antibiotic collateral sensitivity is contingent on the repeatability of evolution. Nat. communications 10, 1-10 (2019). PMCID: PMC6338734.

13. Scarborough, J. A. et al. Identifying states of collateral sensitivity during the evolution of therapeutic resistance in ewing's sarcoma. Iscience 23, 101293 (2020).

14. Dhawan, A. et al. Collateral sensitivity networks reveal evolutionary instability and novel treatment strategies in alk mutated non-small cell lung cancer. Sci. Reports 7, 1-9 (2017). PMCID: PMC5430816.

15. Blount, Z. D., Lenski, R. E. \& Losos, J. B. Contingency and determinism in evolution: Replaying life's tape. Science 362 (2018).

16. Buffa, F., Harris, A., West, C. \& Miller, C. Large meta-analysis of multiple cancers reveals a common, compact and highly prognostic hypoxia metagene. Br. journal cancer 102, 428 (2010).

17. Ritchie, M. E. et al. limma powers differential expression analyses for rna-sequencing and microarray studies. Nucleic acids research 43, e47-e47 (2015).

18. Tusher, V., Tibshirani, R. \& Chu, C. Significance analysis of microarrays applied to ionizing radiation response. Proc. Natl. Acad. Sci. 98, 5116-5121 (2001).

19. Pollard, K. S., Dudoit, S. \& van der Laan, M. J. Multiple testing procedures: the multtest package and applications to genomics. In Bioinformatics and computational biology solutions using $R$ and bioconductor, 249-271 (Springer, 2005).

20. Dhawan, A., Barberis, A., Cheng, W.-C. \& Buffa, F. sigQC: Quality Control Metrics for Gene Signatures (2018). R package version 0.1.21.

21. Dhawan, A. et al. Guidelines for using sigqc for systematic evaluation of gene signatures. Nat. Protoc. 14, 1377 (2019).

22. Azuaje, F. Computational models for predicting drug responses in cancer research. Briefings bioinformatics 18, 820-829 (2017).

23. Goodspeed, A., Heiser, L. M., Gray, J. W. \& Costello, J. C. Tumor-derived cell lines as molecular models of cancer pharmacogenomics. Mol. Cancer Res. 14, 3-13 (2016).

24. Simon, R. M., Paik, S. \& Hayes, D. F. Use of archived specimens in evaluation of prognostic and predictive biomarkers. $J$. Natl. Cancer Inst. 101, 1446-1452 (2009). 
medRxiv preprint doi: https://doi.org/10.1101/2021.11.10.21265799; this version posted November 11, 2021. The copyright holder for this preprint (which was not certified by peer review) is the author/funder, who has granted medRxiv a license to display the preprint in perpetuity.

It is made available under a CC-BY-NC 4.0 International license .

25. Yang, W. et al. Genomics of drug sensitivity in cancer (gdsc): a resource for therapeutic biomarker discovery in cancer cells. Nucleic Acids Res. 41, D955-D961, DOI: 10.1093/nar/gks1111 (2013). /oup/backfile/content_public/journal/nar/41/ d1/10.1093/nar/gks1111/2/gks1111.pdf.

26. Irizarry, R. A. et al. Exploration, normalization, and summaries of high density oligonucleotide array probe level data. Biostatistics 4, 249-264 (2003).

27. Iorio, F. et al. A landscape of pharmacogenomic interactions in cancer. Cell 166, 740-754 (2016).

28. Vis, D. J. et al. Multilevel models improve precision and speed of ic50 estimates. Pharmacogenomics 17, 691-700 (2016).

29. Samur, M. K. Rtcgatoolbox: a new tool for exporting tcga firehose data. PloS one 9, e106397 (2014).

30. Fenstermacher, D. A., Wenham, R. M., Rollison, D. E. \& Dalton, W. S. Implementing personalized medicine in a cancer center. Cancer journal (Sudbury, Mass.) 17, 528 (2011).

31. Dalton, W. S. The "total cancer care" concept: linking technology and health care. Cancer Control. 12, 140-141 (2005).

32. Welsh, E. A., Eschrich, S. A., Berglund, A. E. \& Fenstermacher, D. A. Iterative rank-order normalization of gene expression microarray data. BMC bioinformatics 14, 1-11 (2013).

33. Baccarella, A., Williams, C. R., Parrish, J. Z. \& Kim, C. C. Empirical assessment of the impact of sample number and read depth on rna-seq analysis workflow performance. BMC bioinformatics 19, 423 (2018). 
medRxiv preprint doi: https://doi.org/10.1101/2021.11.10.21265799; this version posted November 11, 2021. The copyright holder for this preprint (which was not certified by peer review) is the author/funder, who has granted medRxiv a license to display the preprint in perpetuity.

It is made available under a CC-BY-NC 4.0 International license.

428

\section{Supplementary Tables}

Table 1. Tissue of origin for all 429 epithelial-origin GDSC cell lines.

\begin{tabular}{ll}
\hline Tissue of origin & No. cell lines. \\
\hline NSCLC adenocarcinoma & 53 \\
Breast & 50 \\
Large intestine & 42 \\
Ovary & 33 \\
Esophagus & 30 \\
Pancreas & 30 \\
Head and neck & 28 \\
Stomach & 25 \\
Bladder & 18 \\
Kidney & 17 \\
Mesothelium & 16 \\
Liver & 15 \\
NSCLC squamous & 15 \\
Cervix & 14 \\
Thyroid & 14 \\
Endometrium & 11 \\
Prostate & 7 \\
Biliary tract & 5 \\
Testis & 3 \\
Uterus & 2 \\
Adrenal gland & 1 \\
\hline
\end{tabular}


medRxiv preprint doi: https://doi.org/10.1101/2021.11.10.21265799; this version posted November 11, 2021. The copyright holder for this preprint (which was not certified by peer review) is the author/funder, who has granted medRxiv a license to display the preprint in perpetuity.

It is made available under a CC-BY-NC 4.0 International license .

Table 2. Tissue of origin for DE comparison groups for fold 1.

\begin{tabular}{|c|c|c|}
\hline Drug Response & Tissue of origin & No. cell lines \\
\hline \multirow{17}{*}{ Resistant } & NSCLC adenocarcinoma & 13 \\
\hline & Pancreas & 9 \\
\hline & Breast & 8 \\
\hline & Large intestine & 7 \\
\hline & Mesothelium & 5 \\
\hline & Kidney & 4 \\
\hline & Ovary & 4 \\
\hline & Stomach & 3 \\
\hline & Thyroid & 3 \\
\hline & Biliary Tract & 2 \\
\hline & Endometrium & 2 \\
\hline & Liver & 2 \\
\hline & NSCLC squamous & 2 \\
\hline & Esophagus & 2 \\
\hline & Cervix & 1 \\
\hline & Head and neck & 1 \\
\hline & Prostate & 1 \\
\hline \multirow{16}{*}{ Sensitive } & Head and neck & 10 \\
\hline & NSCLC adenocarcinoma & 10 \\
\hline & Ovary & 6 \\
\hline & Breast & 5 \\
\hline & Large intestine & 5 \\
\hline & Esophagus & 5 \\
\hline & Bladder & 4 \\
\hline & Kidney & 4 \\
\hline & Pancreas & 4 \\
\hline & Stomach & 4 \\
\hline & Cervix & 3 \\
\hline & Thyroid & 3 \\
\hline & NSCLC squamous & 2 \\
\hline & Prostate & 1 \\
\hline & Testis & 1 \\
\hline & Uterus & 1 \\
\hline
\end{tabular}


medRxiv preprint doi: https://doi.org/10.1101/2021.11.10.21265799; this version posted November 11, 2021. The copyright holder for this preprint (which was not certified by peer review) is the author/funder, who has granted medRxiv a license to display the preprint in perpetuity.

It is made available under a CC-BY-NC 4.0 International license .

Table 3. Tissue of origin for DE comparison groups for fold 2.

\begin{tabular}{|c|c|c|}
\hline Drug Response & Tissue of origin & No. cell lines \\
\hline \multirow{17}{*}{ Resistant } & NSCLC adenocarcinoma & 13 \\
\hline & Breast & 9 \\
\hline & Pancreas & 9 \\
\hline & Large intestine & 5 \\
\hline & Mesothelium & 5 \\
\hline & Stomach & 5 \\
\hline & Esophagus & 4 \\
\hline & Ovary & 4 \\
\hline & Biliary Tract & 2 \\
\hline & Head and neck & 2 \\
\hline & Kidney & 2 \\
\hline & Liver & 2 \\
\hline & NSCLC squamous & 2 \\
\hline & Thyroid & 2 \\
\hline & Bladder & 1 \\
\hline & Endometrium & 1 \\
\hline & Prostate & 1 \\
\hline \multirow{17}{*}{ Sensitive } & Head and neck & 11 \\
\hline & NSCLC adenocarcinoma & 8 \\
\hline & Bladder & 6 \\
\hline & Breast & 5 \\
\hline & Large intestine & 5 \\
\hline & Esophagus & 5 \\
\hline & Ovary & 5 \\
\hline & Stomach & 5 \\
\hline & Cervix & 3 \\
\hline & Kidney & 3 \\
\hline & Pancreas & 3 \\
\hline & NSCLC squamous & 2 \\
\hline & Testis & 2 \\
\hline & Thyroid & 2 \\
\hline & Endometrium & 1 \\
\hline & Prostate & 1 \\
\hline & Uterus & 1 \\
\hline
\end{tabular}


medRxiv preprint doi: https://doi.org/10.1101/2021.11.10.21265799; this version posted November 11, 2021. The copyright holder for this preprint (which was not certified by peer review) is the author/funder, who has granted medRxiv a license to display the preprint in perpetuity.

It is made available under a CC-BY-NC 4.0 International license .

Table 4. Tissue of origin for DE comparison groups for fold 3.

\begin{tabular}{|c|c|c|}
\hline Drug Response & Tissue of origin & No. cell lines \\
\hline \multirow{18}{*}{ Resistant } & NSCLC adenocarcinoma & 13 \\
\hline & Pancreas & 10 \\
\hline & Breast & 8 \\
\hline & Large intestine & 5 \\
\hline & Mesothelium & 5 \\
\hline & Kidney & 4 \\
\hline & Ovary & 4 \\
\hline & Stomach & 4 \\
\hline & Esophagus & 3 \\
\hline & Thyroid & 3 \\
\hline & Endometrium & 2 \\
\hline & Head and neck & 2 \\
\hline & Biliary Tract & 1 \\
\hline & Bladder & 1 \\
\hline & Cervix & 1 \\
\hline & Liver & 1 \\
\hline & NSCLC squamous & 1 \\
\hline & Prostate & 1 \\
\hline \multirow{16}{*}{ Sensitive } & Ovary & 10 \\
\hline & Head and neck & 9 \\
\hline & Bladder & 8 \\
\hline & NSCLC adenocarcinoma & 6 \\
\hline & Esophagus & 5 \\
\hline & Breast & 4 \\
\hline & Cervix & 4 \\
\hline & Kidney & 4 \\
\hline & Pancreas & 4 \\
\hline & Stomach & 4 \\
\hline & Large intestine & 3 \\
\hline & Thyroid & 3 \\
\hline & Testis & 2 \\
\hline & Endometrium & 1 \\
\hline & NSCLC squamous & 1 \\
\hline & Uterus & 1 \\
\hline
\end{tabular}


medRxiv preprint doi: https://doi.org/10.1101/2021.11.10.21265799; this version posted November 11, 2021. The copyright holder for this preprint (which was not certified by peer review) is the author/funder, who has granted medRxiv a license to display the preprint in perpetuity.

It is made available under a CC-BY-NC 4.0 International license .

Table 5. Tissue of origin for DE comparison groups for fold 4.

\begin{tabular}{|c|c|c|}
\hline Drug Response & Tissue of origin & No. cell lines \\
\hline \multirow{17}{*}{ Resistant } & NSCLC adenocarcinoma & 12 \\
\hline & Breast & 10 \\
\hline & Large intestine & 8 \\
\hline & Pancreas & 6 \\
\hline & Mesothelium & 5 \\
\hline & Ovary & 5 \\
\hline & Thyroid & 4 \\
\hline & Kidney & 3 \\
\hline & Liver & 3 \\
\hline & Esophagus & 3 \\
\hline & Biliary Tract & 2 \\
\hline & Head and neck & 2 \\
\hline & Stomach & 2 \\
\hline & Bladder & 1 \\
\hline & Cervix & 1 \\
\hline & Endometrium & 1 \\
\hline & NSCLC squamous & 1 \\
\hline \multirow{17}{*}{ Sensitive } & Head and neck & 9 \\
\hline & NSCLC adenocarcinoma & 8 \\
\hline & Ovary & 8 \\
\hline & Bladder & 6 \\
\hline & Kidney & 5 \\
\hline & Large intestine & 5 \\
\hline & Stomach & 5 \\
\hline & Cervix & 4 \\
\hline & Esophagus & 4 \\
\hline & Pancreas & 3 \\
\hline & Thyroid & 3 \\
\hline & Breast & 2 \\
\hline & Testis & 2 \\
\hline & Endometrium & 1 \\
\hline & NSCLC squamous & 1 \\
\hline & Prostate & 1 \\
\hline & Uterus & 1 \\
\hline
\end{tabular}


medRxiv preprint doi: https://doi.org/10.1101/2021.11.10.21265799; this version posted November 11, 2021. The copyright holder for this preprint (which was not certified by peer review) is the author/funder, who has granted medRxiv a license to display the preprint in perpetuity.

It is made available under a CC-BY-NC 4.0 International license .

Table 6. Tissue of origin for DE comparison groups for fold 5.

\begin{tabular}{|c|c|c|}
\hline Drug Response & Tissue of origin & No. cell lines \\
\hline \multirow{18}{*}{ Resistant } & NSCLC adenocarcinoma & 13 \\
\hline & Pancreas & 10 \\
\hline & Breast & 8 \\
\hline & Large intestine & 8 \\
\hline & Mesothelium & 4 \\
\hline & Esophagus & 4 \\
\hline & Stomach & 4 \\
\hline & Kidney & 3 \\
\hline & Ovary & 3 \\
\hline & Thyroid & 3 \\
\hline & Endometrium & 2 \\
\hline & Biliary Tract & 1 \\
\hline & Bladder & 1 \\
\hline & Cervix & 1 \\
\hline & Head and neck & 1 \\
\hline & Liver & 1 \\
\hline & NSCLC squamous & 1 \\
\hline & Prostate & 1 \\
\hline \multirow{16}{*}{ Sensitive } & Head and neck & 12 \\
\hline & NSCLC adenocarcinoma & 8 \\
\hline & Ovary & 8 \\
\hline & Stomach & 6 \\
\hline & Bladder & 5 \\
\hline & Breast & 5 \\
\hline & Large intestine & 5 \\
\hline & Esophagus & 5 \\
\hline & Kidney & 4 \\
\hline & Cervix & 3 \\
\hline & Pancreas & 2 \\
\hline & Endometrium & 1 \\
\hline & NSCLC squamous & 1 \\
\hline & Prostate & 1 \\
\hline & Testis & 1 \\
\hline & Thyroid & 1 \\
\hline
\end{tabular}


medRxiv preprint doi: https://doi.org/10.1101/2021.11.10.21265799; this version posted November 11, 2021. The copyright holder for this preprint (which was not certified by peer review) is the author/funder, who has granted medRxiv a license to display the preprint in perpetuity.

It is made available under a CC-BY-NC 4.0 International license .

Table 7. DE genes by fold. The SAM method consistently extracts more genes than limma or multtest. The intersection, however, is much smaller than either limma or multtest, showing significant filtering during the intersection step.

\begin{tabular}{llll}
\hline Fold & Method & No. Up-regulated Genes & No. Down-regulated Genes \\
\hline \multirow{4}{*}{1} & SAM & 1979 & 1083 \\
& limma & 181 & 322 \\
& multtest & 219 & 150 \\
& intersection & 59 & 58 \\
\hline \multirow{3}{*}{2} & SAM & 1397 & 853 \\
& limma & 159 & 302 \\
& multtest & 139 & 115 \\
& intersection & 32 & 41 \\
\hline \multirow{4}{*}{3} & SAM & 2290 & 1143 \\
& limma & 176 & 355 \\
& multtest & 247 & 173 \\
& intersection & 58 & 73 \\
\hline \multirow{4}{*}{4} & SAM & 1904 & 1069 \\
& limma & 188 & 263 \\
& multtest & 237 & 147 \\
& intersection & 61 & 42 \\
\hline \multirow{3}{*}{5} & SAM & 566 & 636 \\
& limma & 156 & 221 \\
& multtest & 93 & 87 \\
& intersection & 34 & 28 \\
\hline
\end{tabular}


medRxiv preprint doi: https://doi.org/10.1101/2021.11.10.21265799; this version posted November 11, 2021. The copyright holder for this preprint (which was not certified by peer review) is the author/funder, who has granted medRxiv a license to display the preprint in perpetuity.

It is made available under a CC-BY-NC 4.0 International license .

Table 8. NCCN Guideline versions used for assessing disease-site specific treatment guidelines.

\begin{tabular}{|c|c|c|c|}
\hline $\begin{array}{l}\text { Disease } \\
\text { Site }\end{array}$ & NCCN Guideline Version & Cisplatin Use & Notes for select circumstances \\
\hline ACC & Neuroendocrine and Adrenal Tumors Version & Yes & \\
\hline BLCA & $\begin{array}{l}3.2021 \\
\text { Bladder Cancer Version } 3.2021 .\end{array}$ & Yes & \\
\hline BRCA & Breast Cancer Version 5.2021 & Select circumstances & $\begin{array}{l}\text { Only for recurrent, unresectable } \\
\text { triple negative BRCA with } \\
\text { germline BRCA } 1 / 2 \text { mutation }\end{array}$ \\
\hline CESC & Cervical Cancer Version 1.2021 & Yes & \\
\hline $\mathrm{CHOL}$ & Hepatobiliary Cancers Version 5.2021 & Yes & \\
\hline COAD & Colon Cancer Version 2.2021 & No & \\
\hline ESCA & $\begin{array}{l}\text { Esophageal and Esophagogastric Junction Can- } \\
\text { cers Version } 3.2021\end{array}$ & Yes & \\
\hline HNSC & Head and Neck Cancers Version 3.2021 & Yes & \\
\hline $\mathrm{KICH}$ & Kidney Cancer Version 2.2022 & No & \\
\hline KIRP & Kidney Cancer Version 2.2022 & No & \\
\hline KIRC & Kidney Cancer Version 2.2022 & No & \\
\hline Kidney & Kidney Cancer Version 2.2022 & No & \\
\hline Renal & Kidney Cancer Version 2.2022 & No & \\
\hline $\begin{array}{l}\text { Pelvis } \\
\text { LIHC }\end{array}$ & Hepatobiliary Cancers Version 3.2021 & No & \\
\hline LUAD & Non-Small Cell Lung Cancer Version 5.2021 & Yes & \\
\hline LUSC & Non-Small Cell Lung Cancer Version 5.2021 & Yes & \\
\hline MESO & Malignant Pleural Mesothelioma Version 2.2021 & Yes & \\
\hline OV & Ovarian Cancer/Fallopian Tube Cancer/ Primary & Yes & \\
\hline PAAD & $\begin{array}{l}\text { Peritoneal Cancer Version } 1.2021 \\
\text { Pancreatic Adenocarcinoma Version } 2.2021\end{array}$ & Select circumstances & $\begin{array}{l}\text { Only for BRCA } 1 / 2 \text { or PALB2 } \\
\text { mutations }\end{array}$ \\
\hline PRAD & Prostate Cancer Version 2.2021 & No & \\
\hline READ & Rectal Cancer Version 1.2021 & No & \\
\hline STAD & Gastric cancer Version 3.2021 & Yes & \\
\hline THCA & Thyroid Carcinoma Version 1.2021 & Select circumstances & $\begin{array}{l}\text { Only as adjuvant/radiosensitizer } \\
\text { for anaplastic carcinoma }\end{array}$ \\
\hline THYM & Thymomas and Thymic Carcinomas Version & Yes & \\
\hline UCEC & Uterine Neoplasms Version 3.2021 & Yes & \\
\hline
\end{tabular}


medRxiv preprint doi: https://doi.org/10.1101/2021.11.10.21265799; this version posted November 11, 2021. The copyright holder for this preprint (which was not certified by peer review) is the author/funder, who has granted medRxiv a license to display the preprint in perpetuity.

It is made available under a CC-BY-NC 4.0 International license.

429

\section{Supplementary Figures}

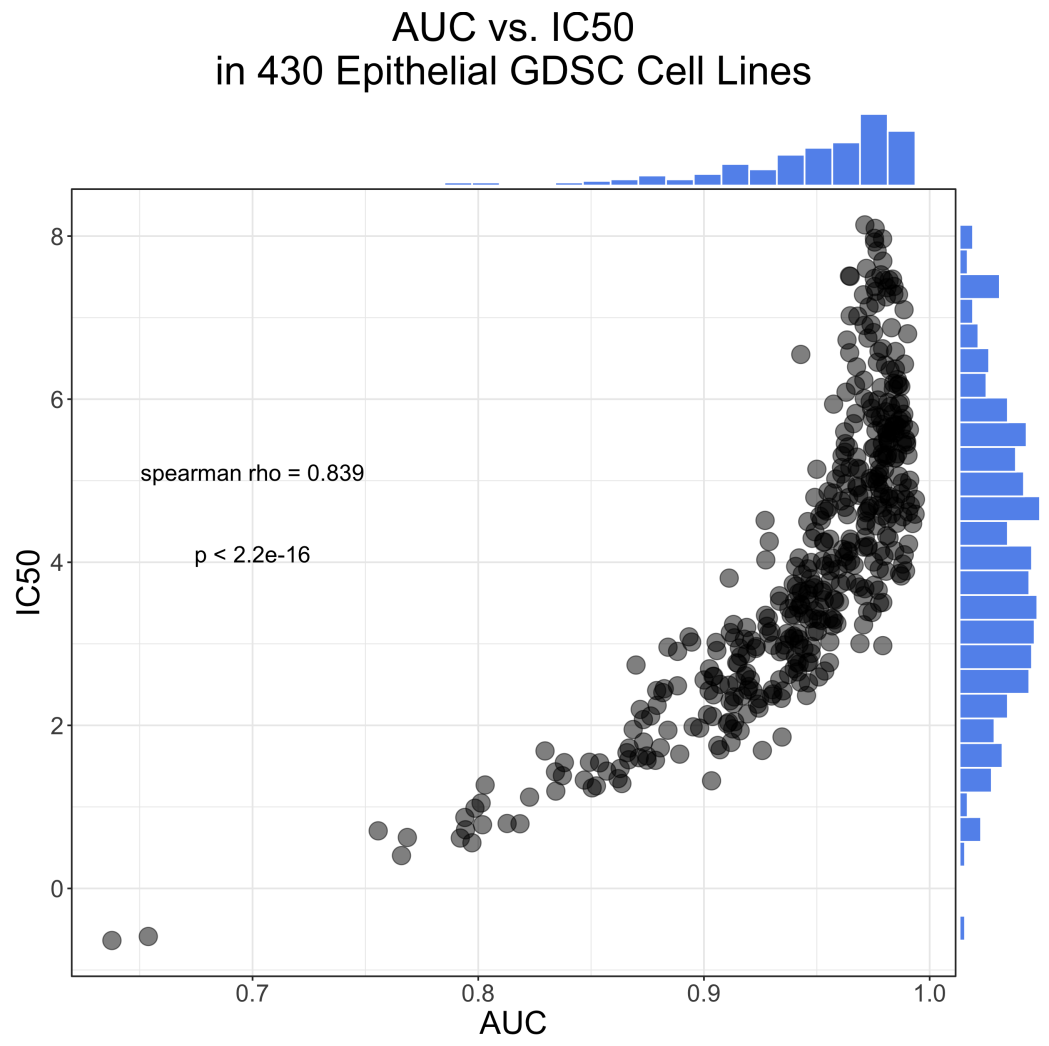

Figure 1. Correlation between AUC and IC50 drug response metrics for epithelial-based cancer cell lines in the Genomics of Drug Discovery in Cancer (GDSC) dataset. 
medRxiv preprint doi: https://doi.org/10.1101/2021.11.10.21265799; this version posted November 11, 2021. The copyright holder for this preprint (which was not certified by peer review) is the author/funder, who has granted medRxiv a license to display the preprint in perpetuity.

It is made available under a CC-BY-NC 4.0 International license .

Relative Med. SD

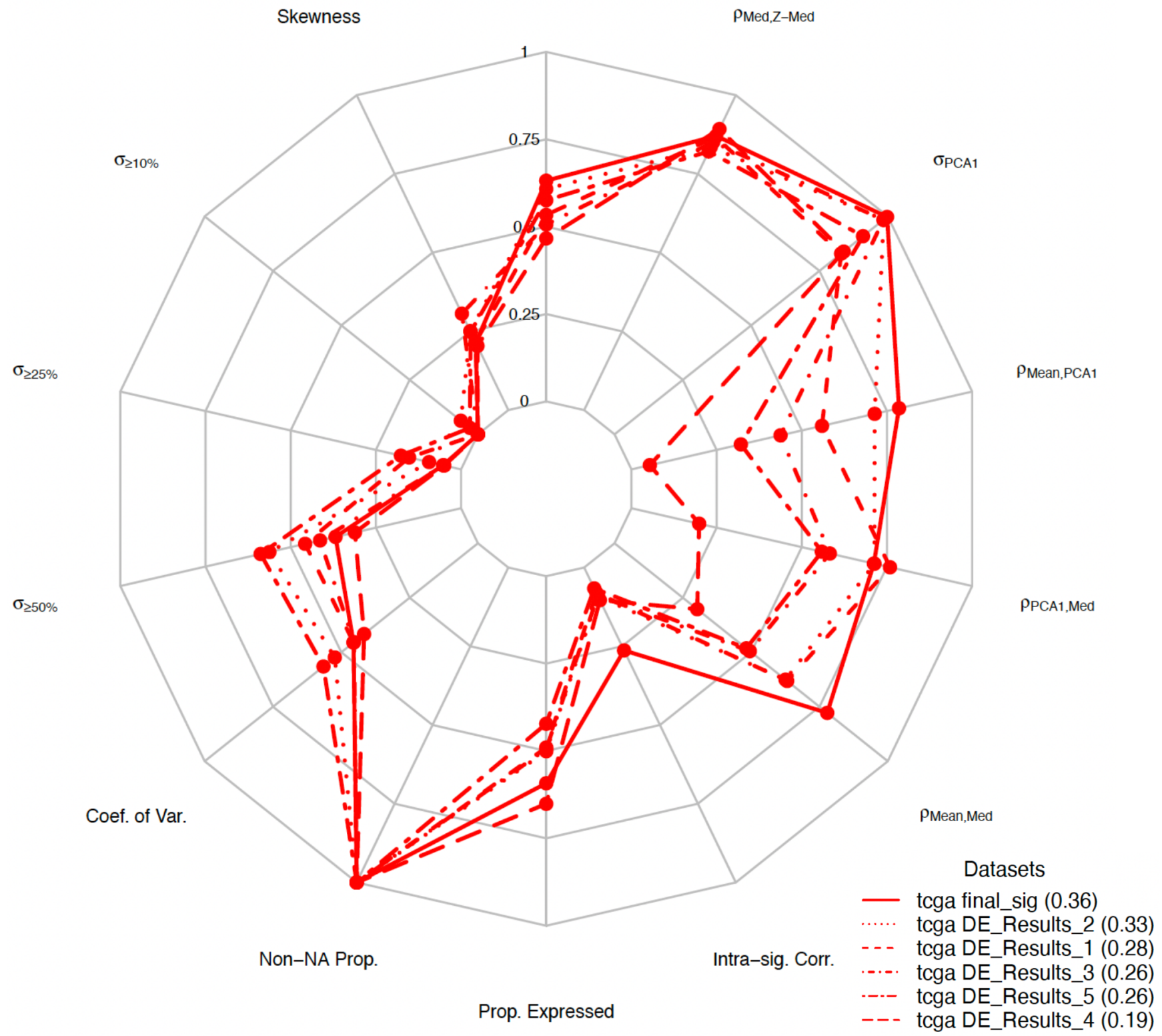

Figure 2. Quality control metrics comparing differential expression results to the final gene signature using sigQC ${ }^{20,21}$. CisSig is compared to the folds of differential gene expression analysis, comparing results using a radar plot. It shows greater intra-signature correlation, higher correlation between signature mean and median, and decreased skewness within RNA-seq expression from TCGA samples of epithelial origin. 
medRxiv preprint doi: https://doi.org/10.1101/2021.11.10.21265799; this version posted November 11, 2021. The copyright holder for this preprint (which was not certified by peer review) is the author/funder, who has granted medRxiv a license to display the preprint in perpetuity.

It is made available under a CC-BY-NC 4.0 International license .

A

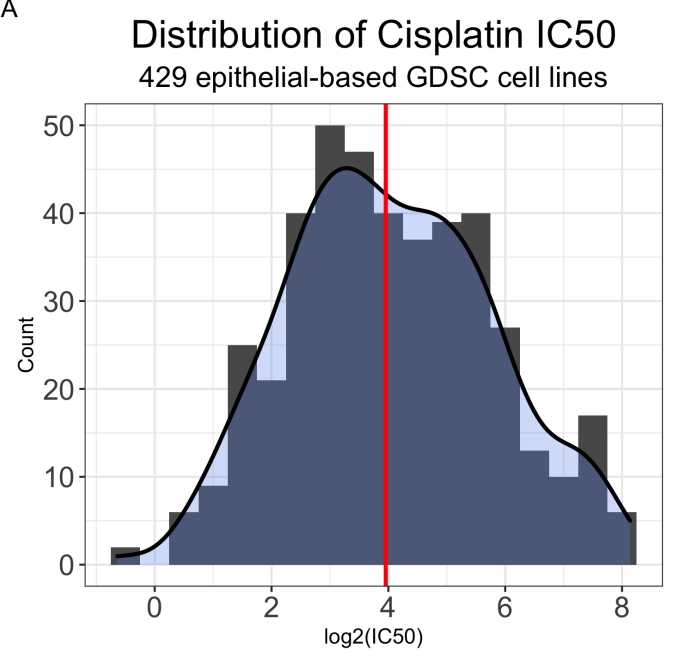

B

Distribution of Signature Scores 429 epithelial-based GDSC cell lines

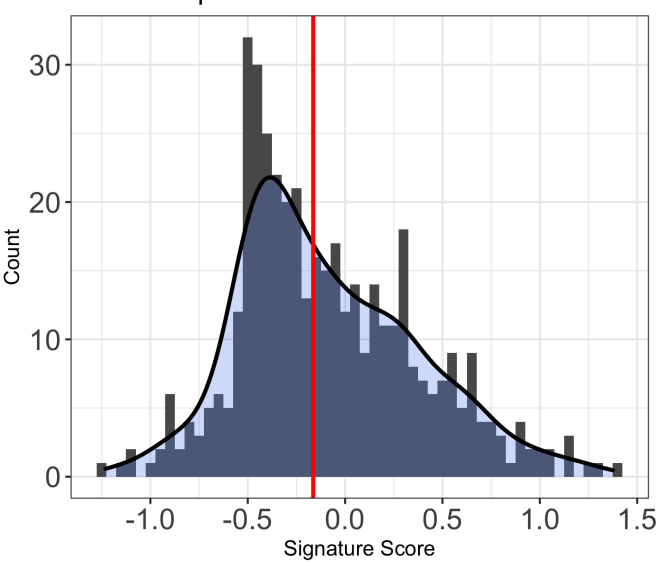

Figure 3. Cisplatin IC50 (log2-transformed) in epithelial-origin GDSC cell lines is relatively normally distributed, while CisSig Score has a right skew. A. Distribution of CisSig across 429 epithelial-based GDSC cell lines, using a histogram (gray) and kernel density estimation (blue). Median score marked by red vertical line. CisSig score is calculated as a cell line's median normalized expression of CisSig genes listed in A. B. Distribution of cisplatin IC50 across 429 epithelial-based GDSC cell lines, using a histogram (gray) and kernel density estimation (blue). Median IC50 marked by red vertical line. 
medRxiv preprint doi: https://doi.org/10.1101/2021.11.10.21265799; this version posted November 11, 2021. The copyright holder for this preprint (which was not certified by peer review) is the author/funder, who has granted medRxiv a license to display the preprint in perpetuity.

It is made available under a CC-BY-NC 4.0 International license .
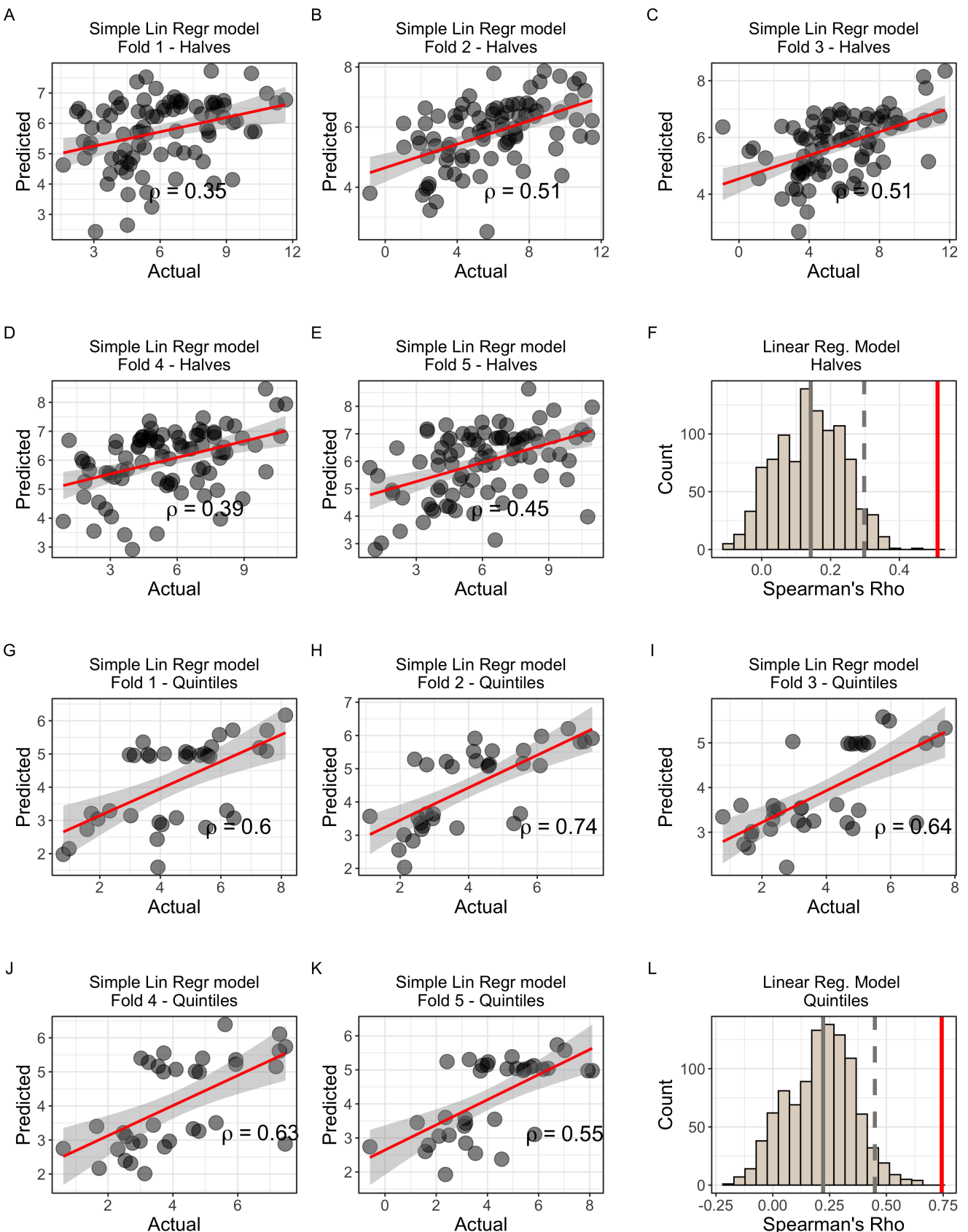

Figure 4. Modeling IC50 response using CisSig Score to predict IC50 in GDSC with simple linear regression. A-E. Predicted vs. Actual IC50 for validation sets of folds 1-5 for models built with all 429 cell lines. F. Null distribution of modeling metrics using 1000 random gene signatures with the same length as CisSig and the model described in A-E. CisSig's performance (red solid line) is within the top 5\% of the null distribution (cutoff at gray dashed line). Gray solid line represents median of null distribution. G-K. Predicted vs. Actual IC50 for validation sets of folds 1-5 for models built using cell lines in the top and bottom $20 \%$ of cisplatin IC50. F. Null distribution of modeling metrics using 1000 random gene signatures with the same length as CisSig and the model described in G-K. CisSig's performance (red solid line) is compared to the 95\% confidence interval (gray dashed line) of the null distribution. Gray solid line represents median of null distribution. 
medRxiv preprint doi: https://doi.org/10.1101/2021.11.10.21265799; this version posted November 11, 2021. The copyright holder for this preprint (which was not certified by peer review) is the author/funder, who has granted medRxiv a license to display the preprint in perpetuity.

It is made available under a CC-BY-NC 4.0 International license .
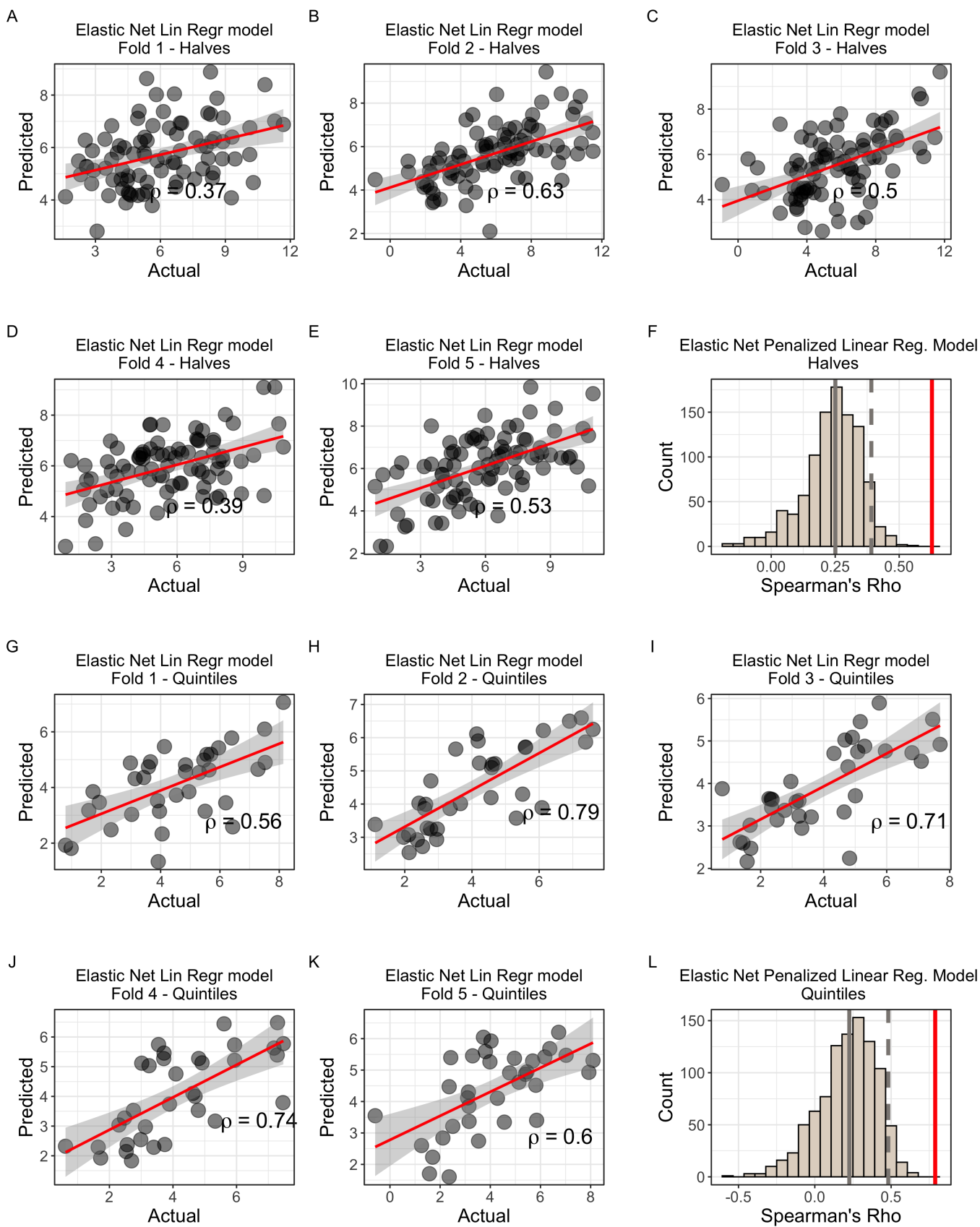

Figure 5. Modeling IC50 response using individual CisSig genes to predict IC50 in GDSC with elastic net penalized linear regression. A-E. Predicted vs. Actual IC50 for validation sets of folds 1-5 for models built with all 429 cell lines. F. Null distribution of modeling metrics using 1000 random gene signatures with the same length as CisSig and the model described in A-E. CisSig's performance (red solid line) is within the top 5\% of the null distribution (cutoff at gray dashed line). Gray solid line represents median of null distribution. G-K. Predicted vs. Actual IC50 for validation sets of folds 1-5 for models built using cell lines in the top and bottom $20 \%$ of cisplatin IC50. F. Null distribution of modeling metrics using 1000 random gene signatures with the same length as CisSig and the model described in G-K. CisSig's performance (red solid line) is compared to the $95 \%$ confidence interval (gray dashed line) of the null distribution. Gray solid line represents median of null distribution. 
medRxiv preprint doi: https://doi.org/10.1101/2021.11.10.21265799; this version posted November 11, 2021. The copyright holder for this preprint (which was not certified by peer review) is the author/funder, who has granted medRxiv a license to display the preprint in perpetuity.

It is made available under a CC-BY-NC 4.0 International license .
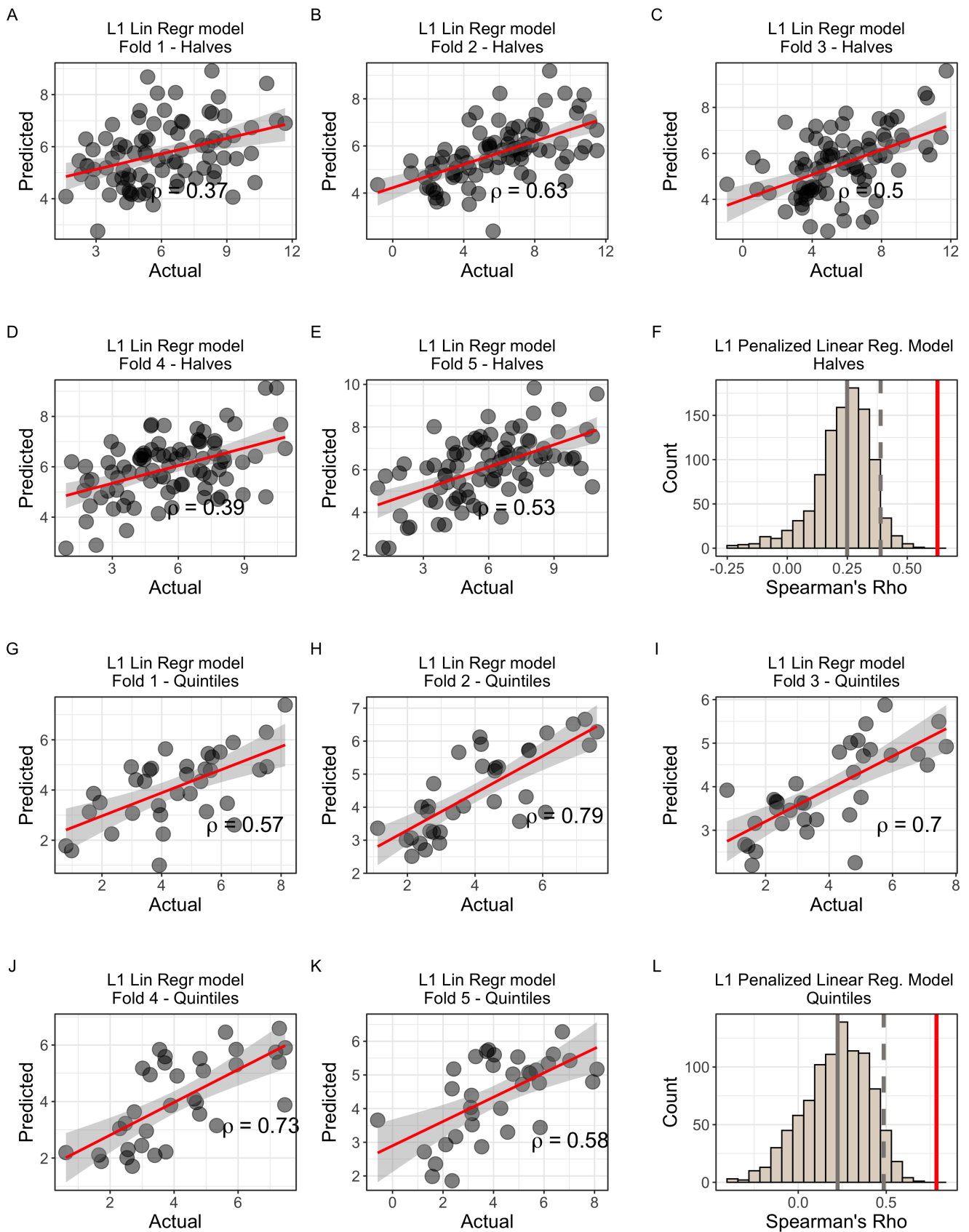

Figure 6. Modeling IC50 response using individual CisSig genes to predict IC50 in GDSC with L1 penalized linear regression. A-E. Predicted vs. Actual IC50 for validation sets of folds 1-5 for models built with all 429 cell lines. F. Null distribution of modeling metrics using 1000 random gene signatures with the same length as CisSig and the model described in A-E. CisSig's performance (red solid line) is within the top 5\% of the null distribution (cutoff at gray dashed line). Gray solid line represents median of null distribution. G-K. Predicted vs. Actual IC50 for validation sets of folds 1-5 for models built using cell lines in the top and bottom $20 \%$ of cisplatin IC50. F. Null distribution of modeling metrics using 1000 random gene signatures with the same length as CisSig and the model described in G-K. CisSig's performance (red solid line) is compared to the $95 \%$ confidence interval (gray dashed line) of the null distribution. Gray solid line represents median of null distribution. 
medRxiv preprint doi: https://doi.org/10.1101/2021.11.10.21265799; this version posted November 11, 2021. The copyright holder for this preprint (which was not certified by peer review) is the author/funder, who has granted medRxiv a license to display the preprint in perpetuity.

It is made available under a CC-BY-NC 4.0 International license .
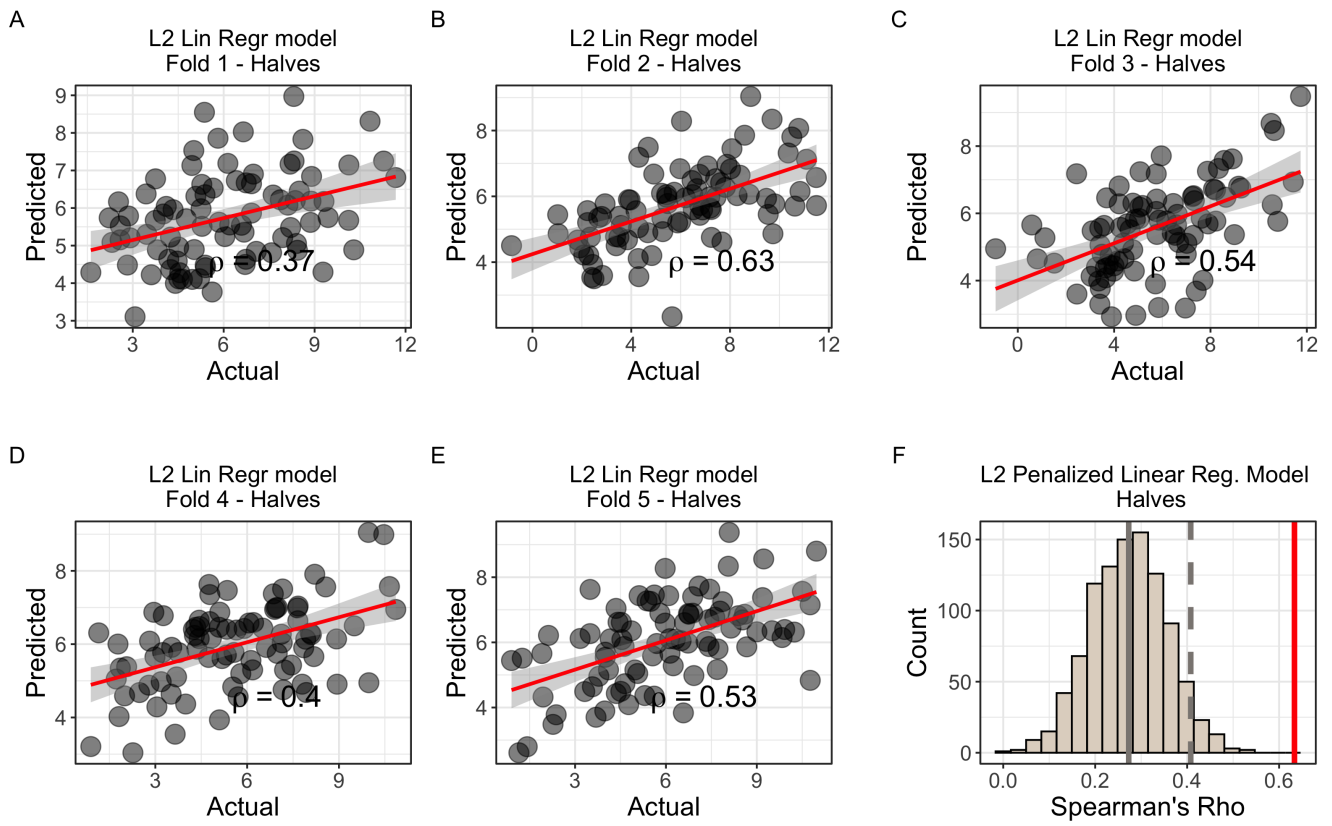

F
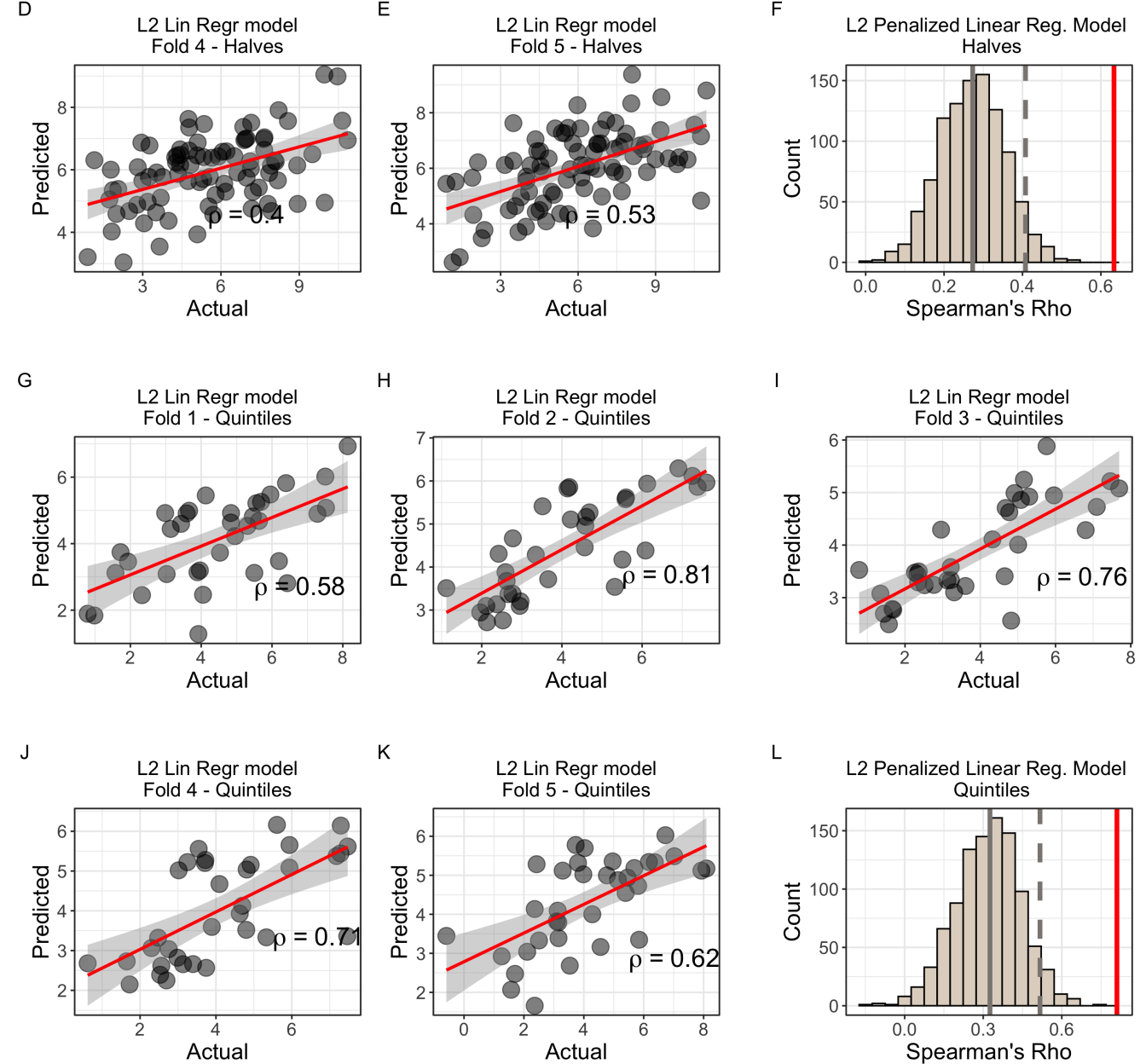

Figure 7. Modeling IC50 response using individual CisSig genes to predict IC50 in GDSC with L2 penalized linear regression. A-E. Predicted vs. Actual IC50 for validation sets of folds 1-5 for models built with all 429 cell lines. F. Null distribution of modeling metrics using 1000 random gene signatures with the same length as CisSig and the model described in A-E. CisSig's performance (red solid line) is within the top 5\% of the null distribution (cutoff at gray dashed line). Gray solid line represents median of null distribution. G-K. Predicted vs. Actual IC50 for validation sets of folds 1-5 for models built using cell lines in the top and bottom $20 \%$ of cisplatin IC50. F. Null distribution of modeling metrics using 1000 random gene signatures with the same length as CisSig and the model described in G-K. CisSig's performance (red solid line) is compared to the $95 \%$ confidence interval (gray dashed line) of the null distribution. Gray solid line represents median of null distribution. 
A

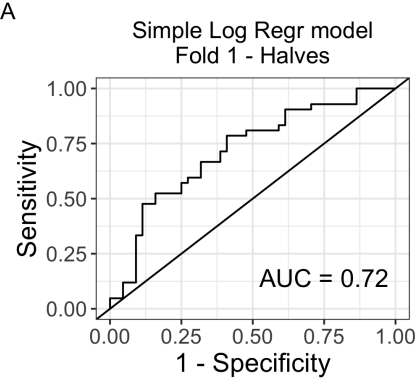

D

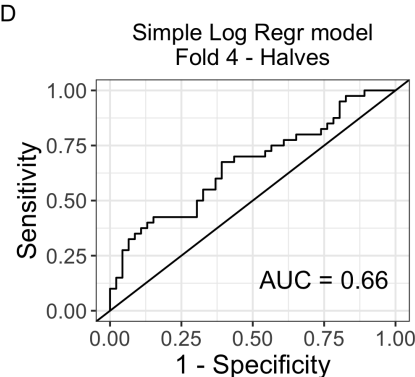

G

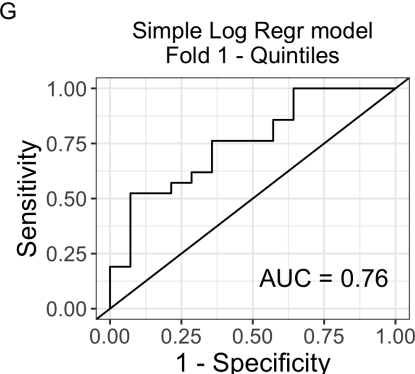

J

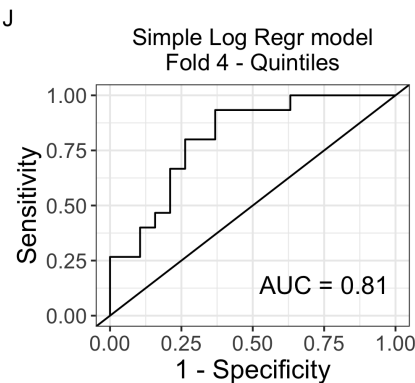

B

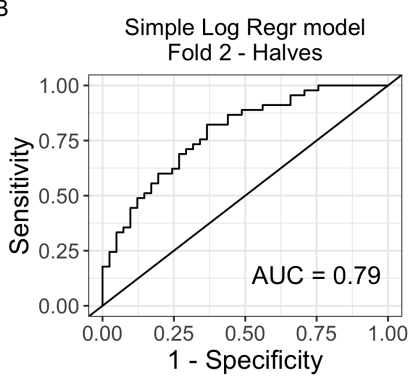

E

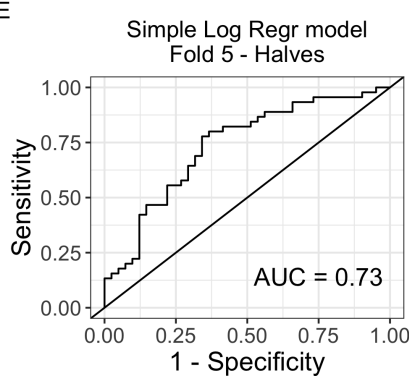

$\mathrm{H}$

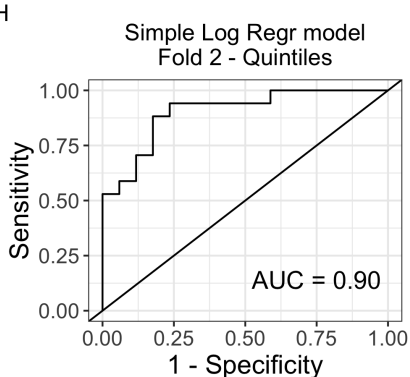

K

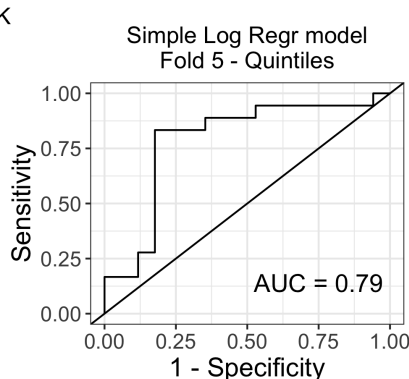

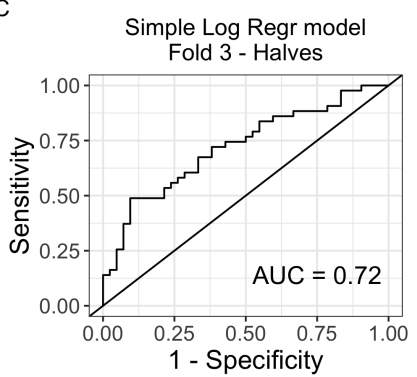

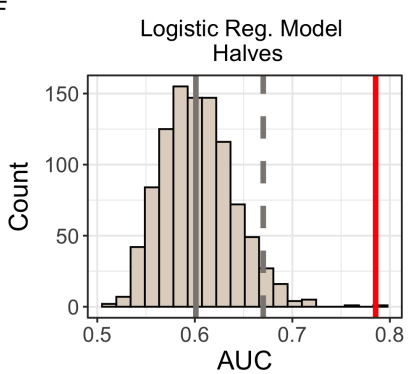

I

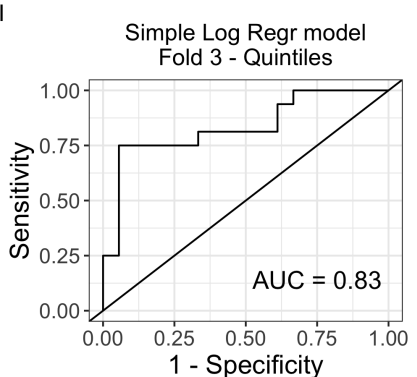

L

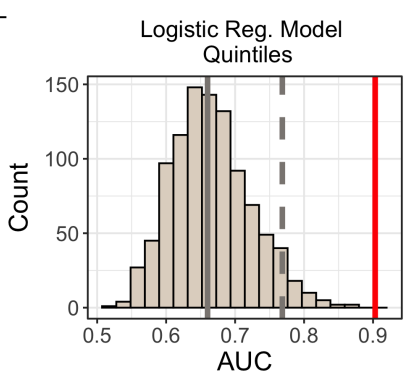

Figure 8. Modeling IC50 response using CisSig score to predict IC50 class in GDSC with simple logistic regression. A-E. Predicted vs. Actual IC50 for validation sets of folds 1-5 for models built with all 429 cell lines. F. Null distribution of modeling metrics using 1000 random gene signatures with the same length as CisSig and the model described in A-E. CisSig's performance (red solid line) is within the top 5\% of the null distribution (cutoff at gray dashed line). Gray solid line represents median of null distribution. G-K. Predicted vs. Actual IC50 for validation sets of folds 1-5 for models built using cell lines in the top and bottom $20 \%$ of cisplatin IC50. F. Null distribution of modeling metrics using 1000 random gene signatures with the same length as CisSig and the model described in G-K. CisSig's performance (red solid line) is compared to the 95\% confidence interval (gray dashed line) of the null distribution. Gray solid line represents median of null distribution. 
A

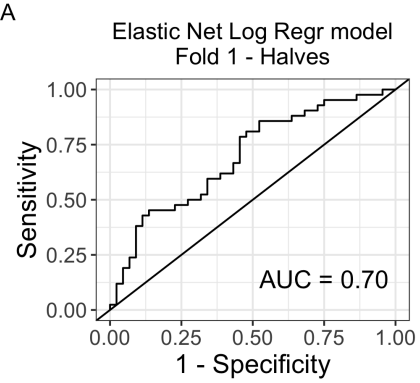

D

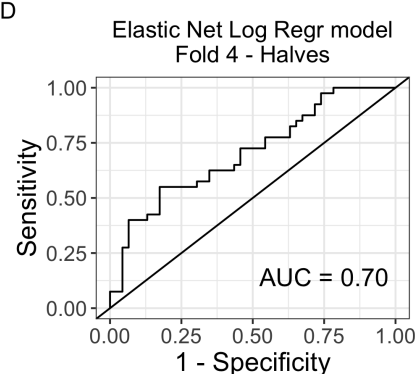

G

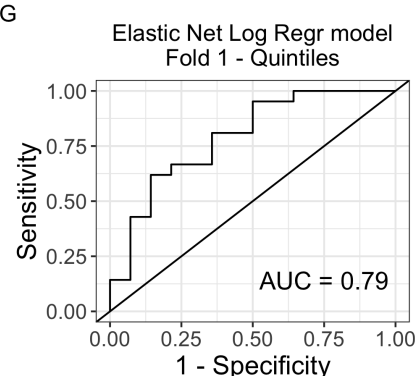

J

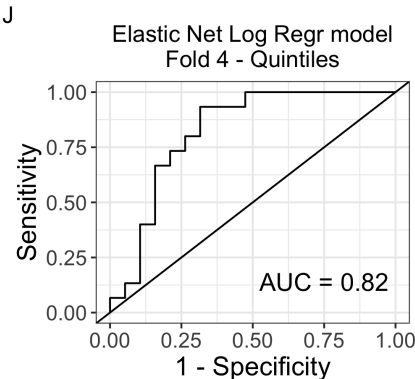

B

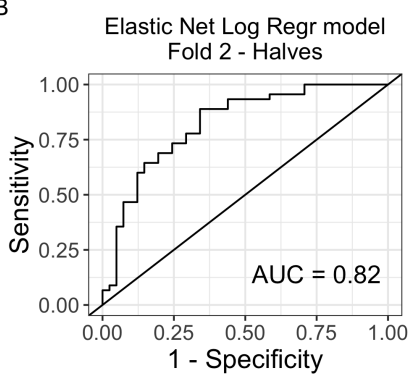

$\mathrm{E}$

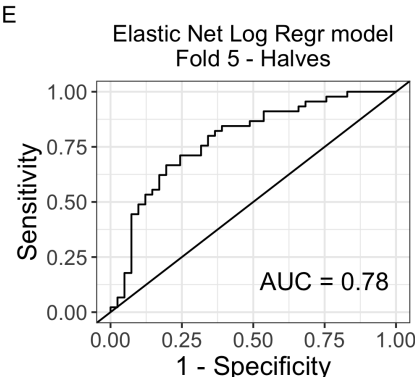

$\mathrm{H}$

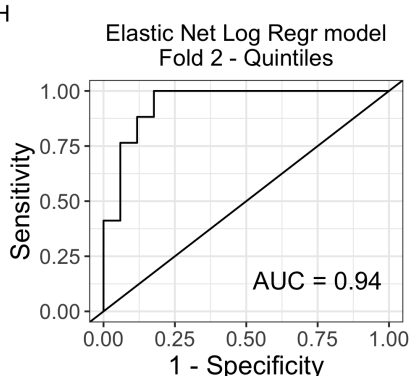

K

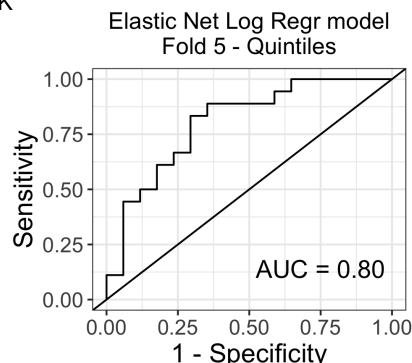

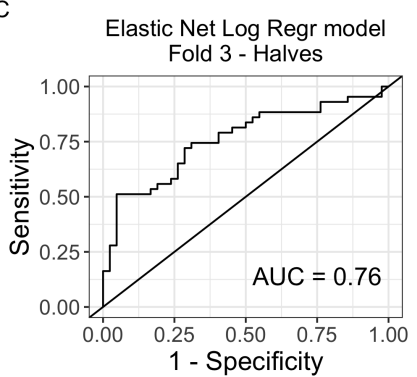

Elastic Net Penalized Logistic Reg. Model
Halves

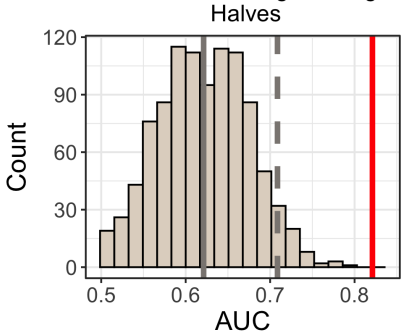

I

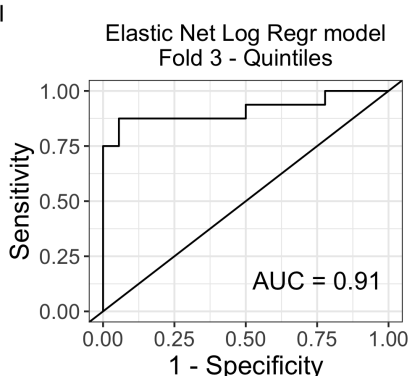

L

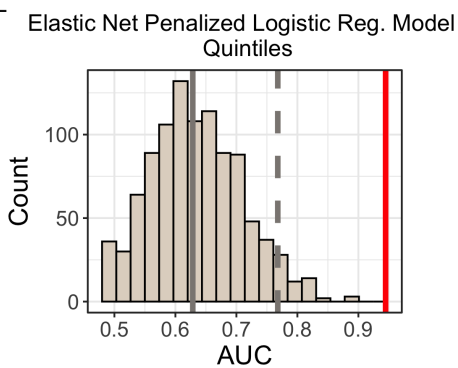

Figure 9. Modeling IC50 response using individual CisSig genes to predict IC50 class in GDSC with elastic net penalized logistic regression. A-E. Predicted vs. Actual IC50 for validation sets of folds 1-5 for models built with all 429 cell lines. F. Null distribution of modeling metrics using 1000 random gene signatures with the same length as CisSig and the model described in A-E. CisSig's performance (red solid line) is within the top 5\% of the null distribution (cutoff at gray dashed line). Gray solid line represents median of null distribution. G-K. Predicted vs. Actual IC50 for validation sets of folds 1-5 for models built using cell lines in the top and bottom $20 \%$ of cisplatin IC50. F. Null distribution of modeling metrics using 1000 random gene signatures with the same length as CisSig and the model described in G-K. CisSig's performance (red solid line) is compared to the $95 \%$ confidence interval (gray dashed line) of the null distribution. Gray solid line represents median of null distribution. 
A

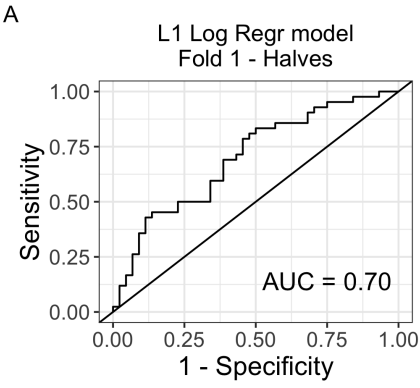

D

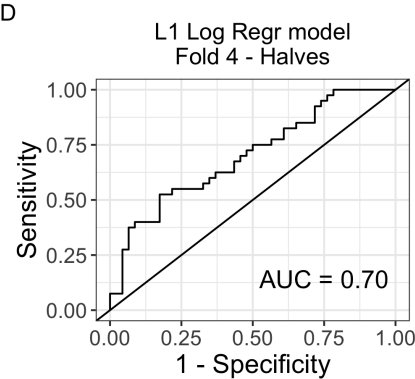

G

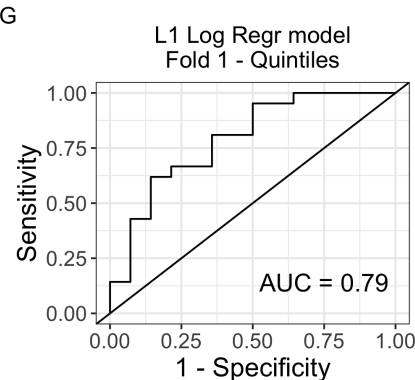

J

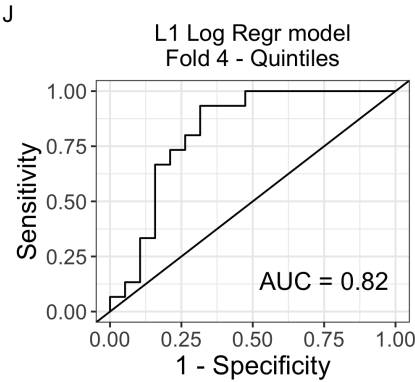

B

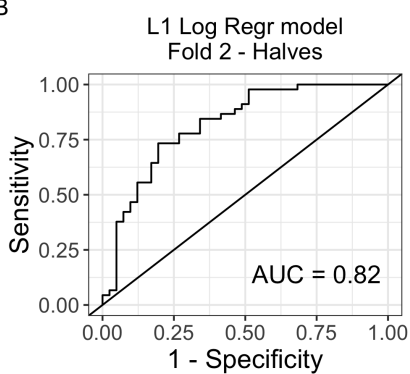

$E$

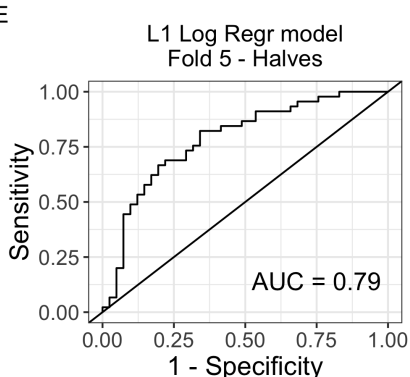

$\mathrm{H}$

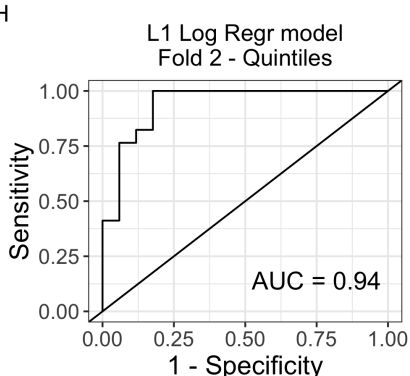

$\mathrm{K}$

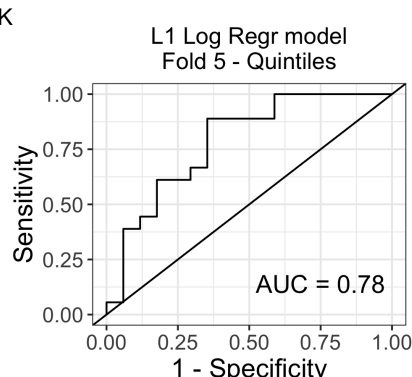

C

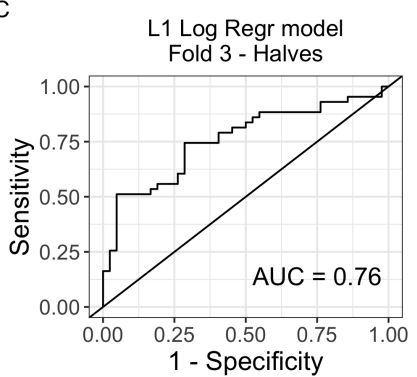

L1 Penalized Logistic Reg. Model

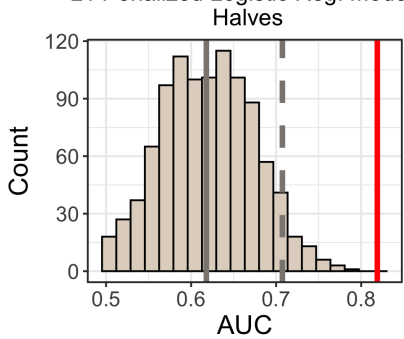

I

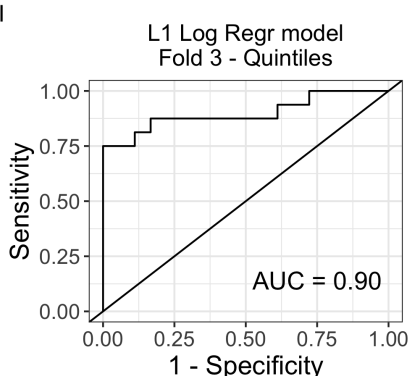

$\mathrm{L}$

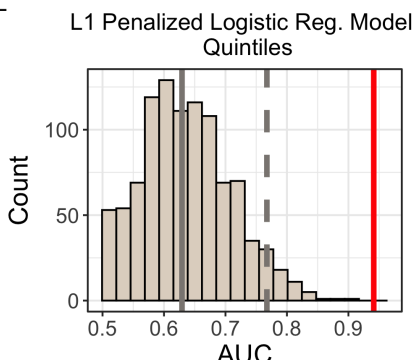

Figure 10. Modeling IC50 response using individual CisSig genes to predict IC50 class in GDSC with L1 penalized logistic regression. A-E. Predicted vs. Actual IC50 for validation sets of folds 1-5 for models built with all 429 cell lines. F. Null distribution of modeling metrics using 1000 random gene signatures with the same length as CisSig and the model described in A-E. CisSig's performance (red solid line) is within the top 5\% of the null distribution (cutoff at gray dashed line). Gray solid line represents median of null distribution. G-K. Predicted vs. Actual IC50 for validation sets of folds 1-5 for models built using cell lines in the top and bottom $20 \%$ of cisplatin IC50. F. Null distribution of modeling metrics using 1000 random gene signatures with the same length as CisSig and the model described in G-K. CisSig's performance (red solid line) is compared to the $95 \%$ confidence interval (gray dashed line) of the null distribution. Gray solid line represents median of null distribution. 
A

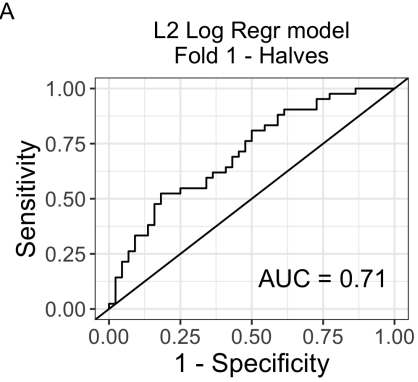

D

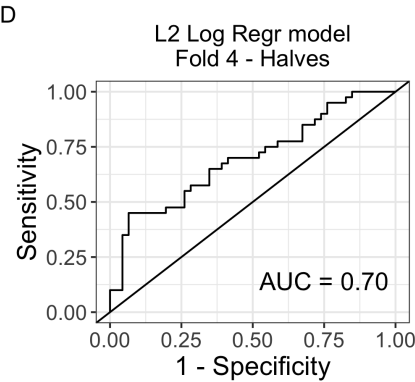

G

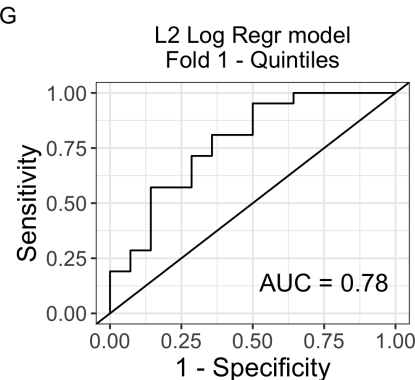

J

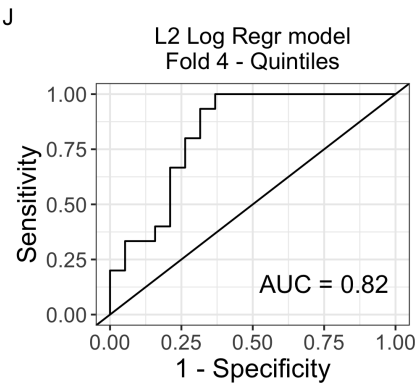

B

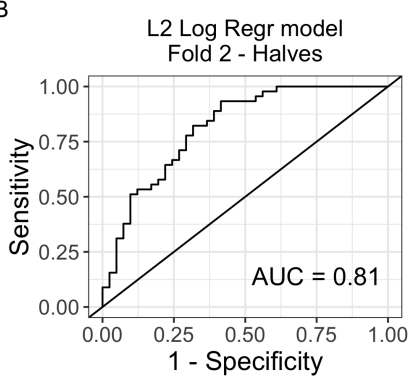

E

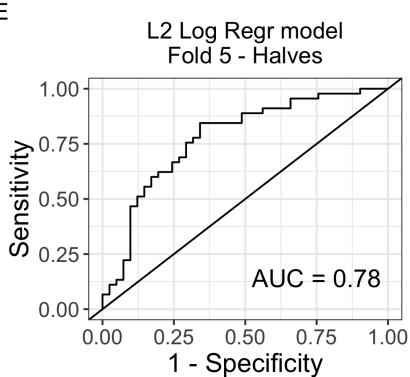

$\mathrm{H}$

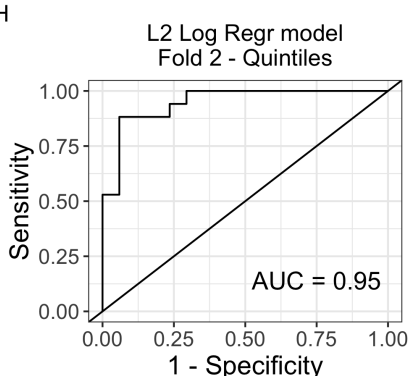

$\mathrm{K}$

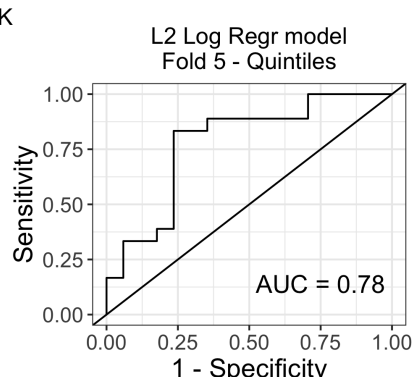

C

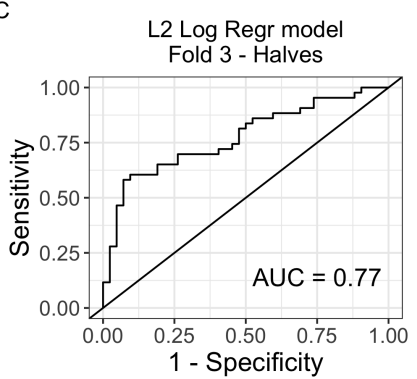

L2 Penalized Logistic Reg. Model

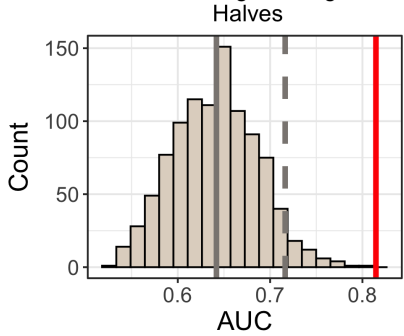

।

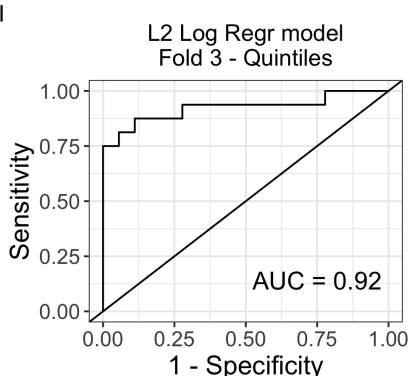

L

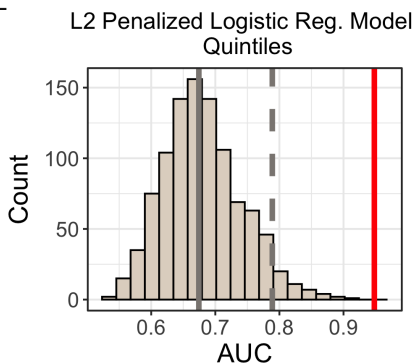

Figure 11. Modeling IC50 response using individual CisSig genes to predict IC50 class in GDSC with L2 penalized logistic regression. A-E. Predicted vs. Actual IC50 for validation sets of folds 1-5 for models built with all 429 cell lines. F. Null distribution of modeling metrics using 1000 random gene signatures with the same length as CisSig and the model described in A-E. CisSig's performance (red solid line) is within the top 5\% of the null distribution (cutoff at gray dashed line). Gray solid line represents median of null distribution. G-K. Predicted vs. Actual IC50 for validation sets of folds 1-5 for models built using cell lines in the top and bottom $20 \%$ of cisplatin IC50. F. Null distribution of modeling metrics using 1000 random gene signatures with the same length as CisSig and the model described in G-K. CisSig's performance (red solid line) is compared to the $95 \%$ confidence interval (gray dashed line) of the null distribution. Gray solid line represents median of null distribution. 
A

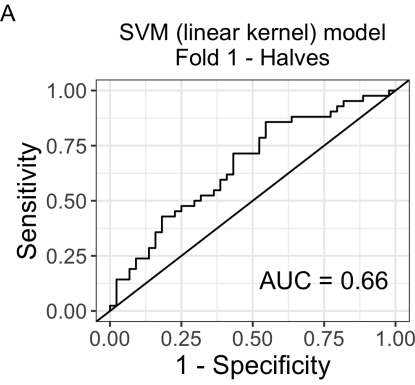

D

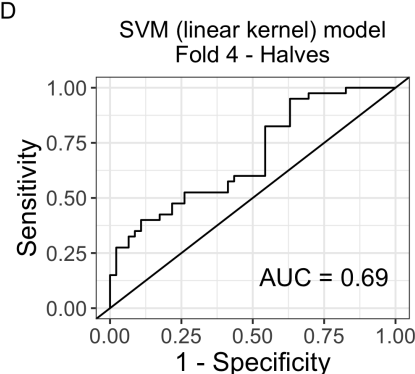

G

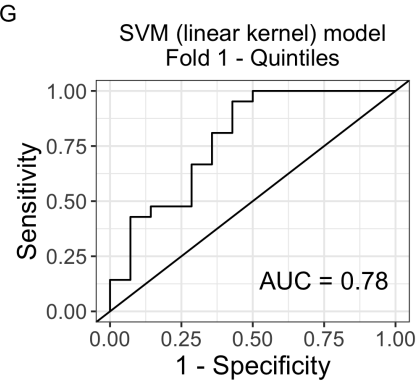

J

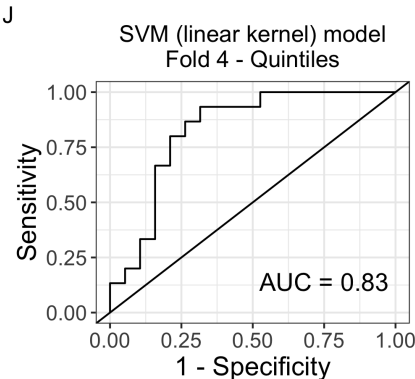

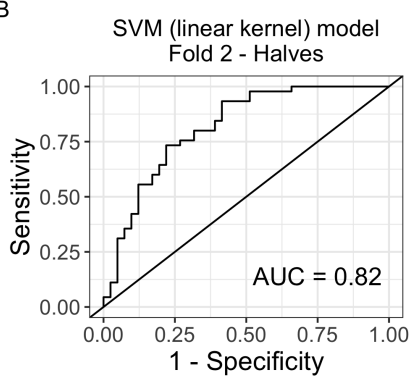

E

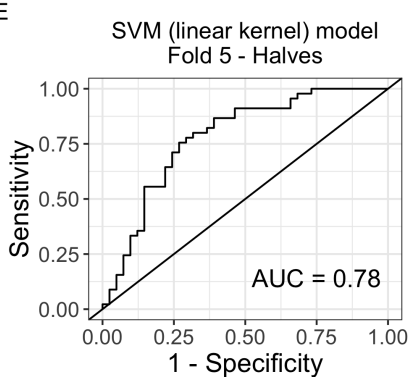

$\mathrm{H}$

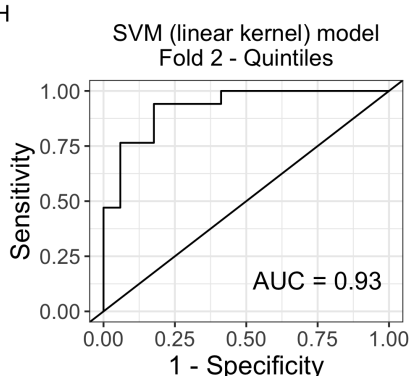

K

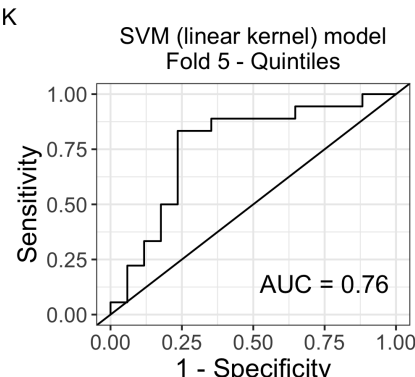

C
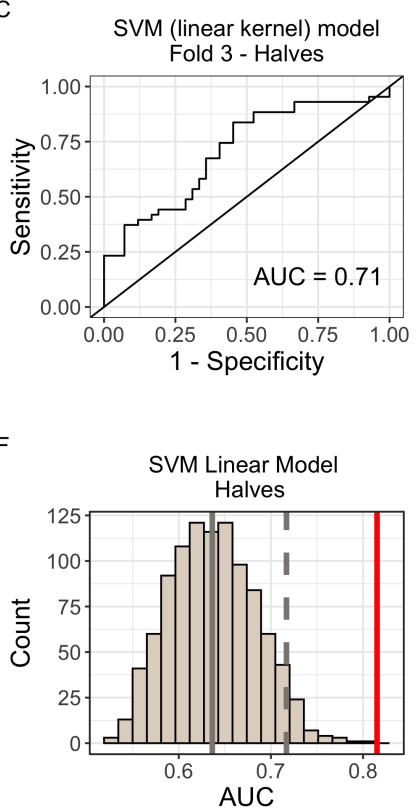

।

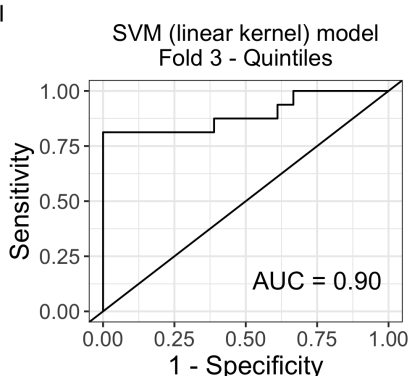

L

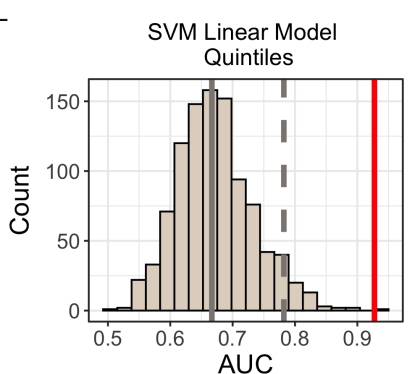

Figure 12. Modeling IC50 response using individual CisSig genes to predict IC50 class in GDSC with support vector machine modeling (linear kernel). A-E. Predicted vs. Actual IC50 for validation sets of folds 1-5 for models built with all 429 cell lines. F. Null distribution of modeling metrics using 1000 random gene signatures with the same length as CisSig and the model described in A-E. CisSig's performance (red solid line) is within the top 5\% of the null distribution (cutoff at gray dashed line). Gray solid line represents median of null distribution. G-K. Predicted vs. Actual IC50 for validation sets of folds 1-5 for models built using cell lines in the top and bottom $20 \%$ of cisplatin IC50. F. Null distribution of modeling metrics using 1000 random gene signatures with the same length as CisSig and the model described in G-K. CisSig's performance (red solid line) is compared to the $95 \%$ confidence interval (gray dashed line) of the null distribution. Gray solid line represents median of null distribution. 
A

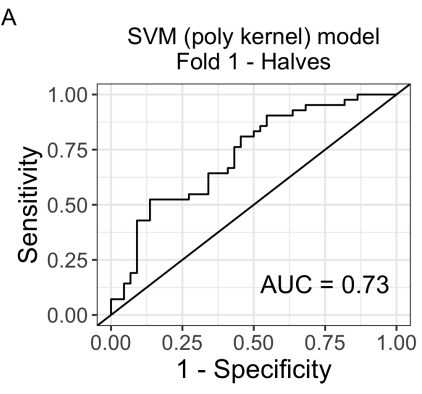

D

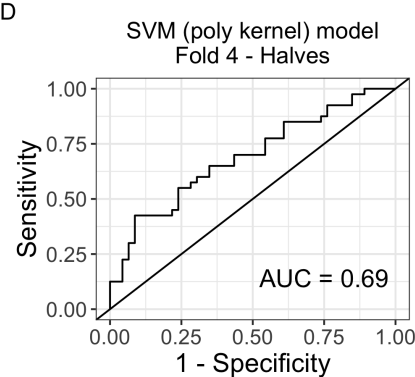

G

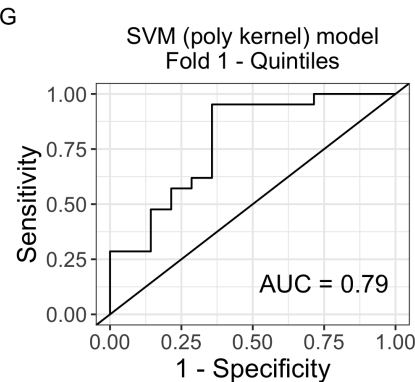

J

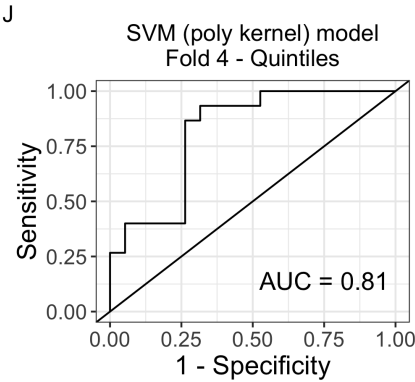

B

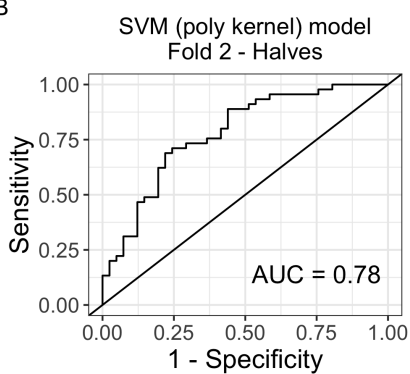

E

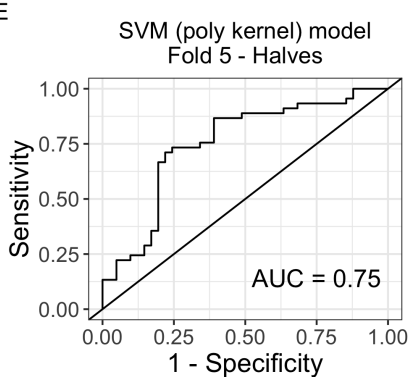

H

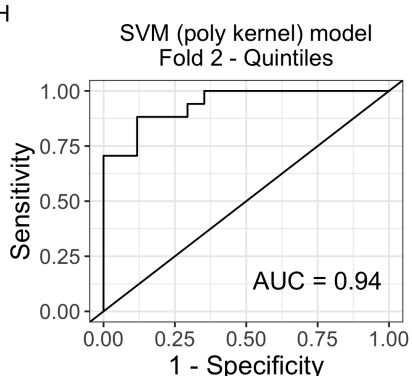

K

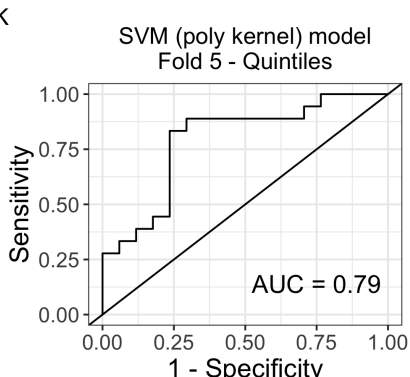

C
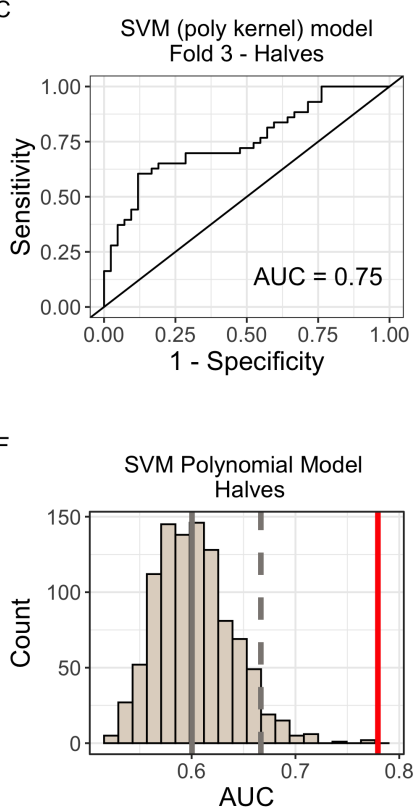

।

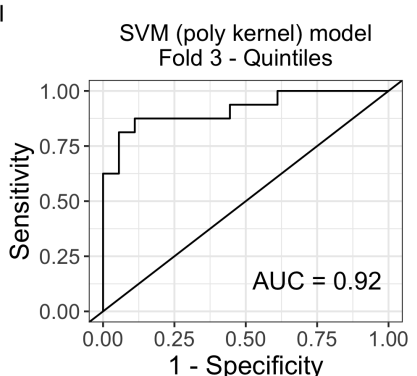

L

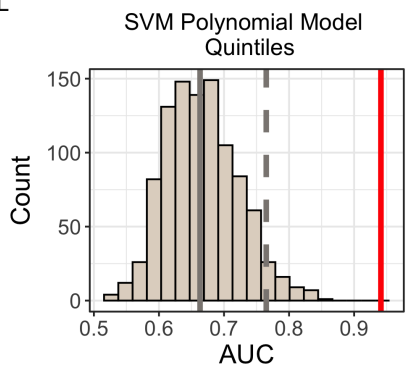

Figure 13. Modeling IC50 response using individual CisSig genes to predict IC50 class in GDSC with support vector machine modeling (polynomial kernel). A-E. Predicted vs. Actual IC50 for validation sets of folds 1-5 for models built with all 429 cell lines. F. Null distribution of modeling metrics using 1000 random gene signatures with the same length as CisSig and the model described in A-E. CisSig's performance (red solid line) is within the top 5\% of the null distribution (cutoff at gray dashed line). Gray solid line represents median of null distribution. G-K. Predicted vs. Actual IC50 for validation sets of folds 1-5 for models built using cell lines in the top and bottom $20 \%$ of cisplatin IC50. F. Null distribution of modeling metrics using 1000 random gene signatures with the same length as CisSig and the model described in G-K. CisSig's performance (red solid line) is compared to the $95 \%$ confidence interval (gray dashed line) of the null distribution. Gray solid line represents median of null distribution. 
A

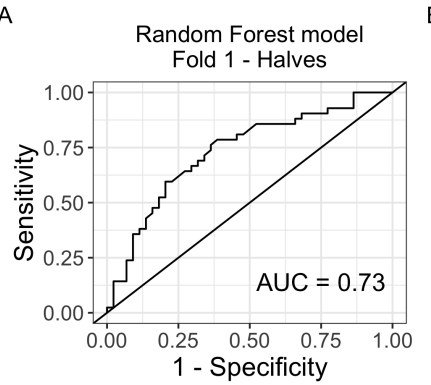

D

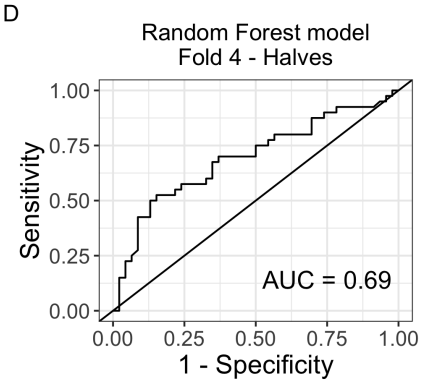

G

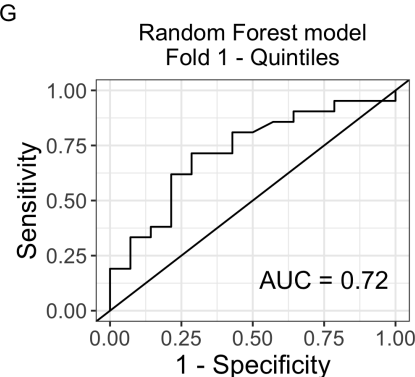

J

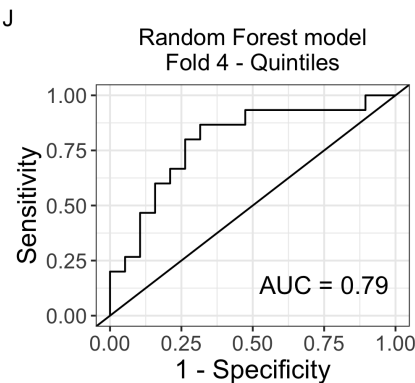

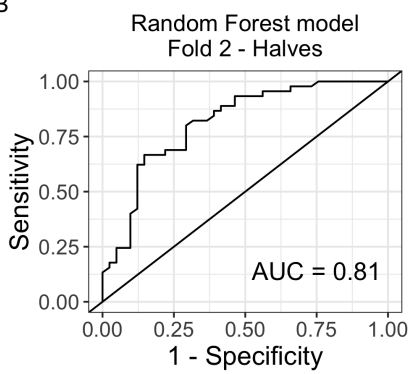

$\mathrm{E}$

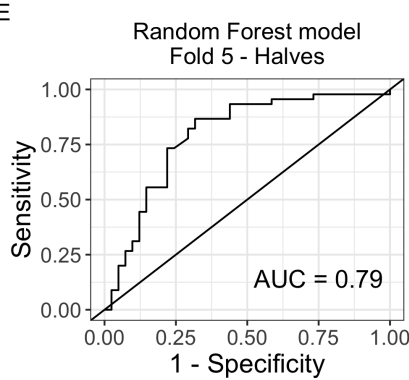

$\mathrm{H}$

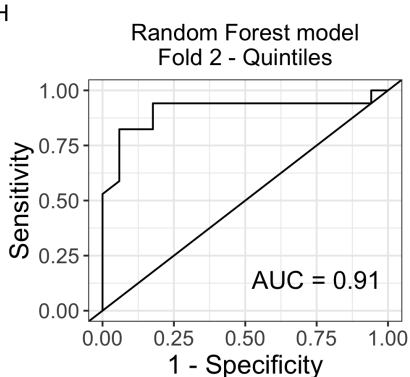

$\mathrm{K}$

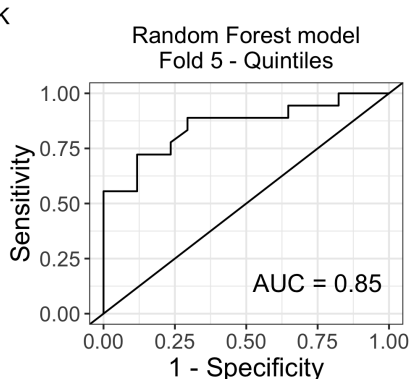

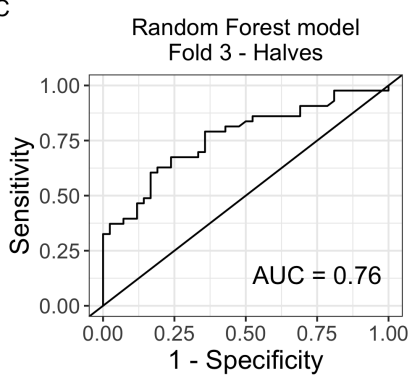

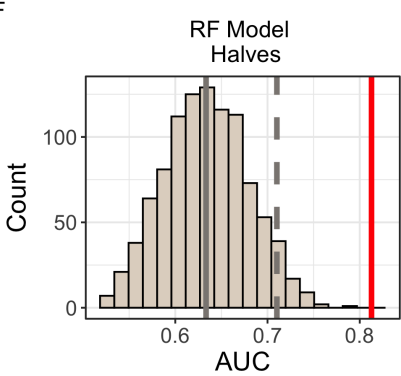

।

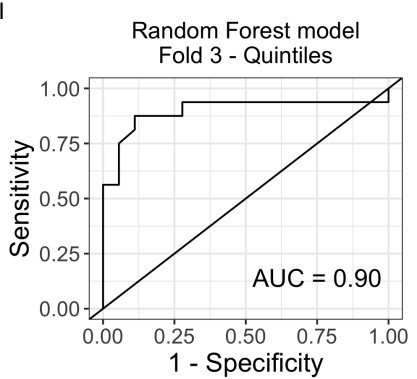

L

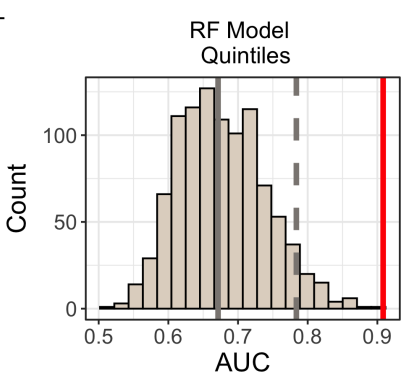

Figure 14. Modeling IC50 response using individual CisSig genes to predict IC50 class in GDSC with random forest modeling. A-E. Predicted vs. Actual IC50 for validation sets of folds 1-5 for models built with all 429 cell lines. F. Null distribution of modeling metrics using 1000 random gene signatures with the same length as CisSig and the model described in A-E. CisSig's performance (red solid line) is within the top 5\% of the null distribution (cutoff at gray dashed line). Gray solid line represents median of null distribution. G-K. Predicted vs. Actual IC50 for validation sets of folds 1-5 for models built using cell lines in the top and bottom $20 \%$ of cisplatin IC50. F. Null distribution of modeling metrics using 1000 random gene signatures with the same length as CisSig and the model described in G-K. CisSig's performance (red solid line) is compared to the $95 \%$ confidence interval (gray dashed line) of the null distribution. Gray solid line represents median of null distribution. 